LA W RENCE LIVERMORE NATIONAL LABORATORY
Advanced discrete-continuum methods for dynamic loading of tunnels by ground shocks

O. Vorobiev

April 4, 2013 
This document was prepared as an account of work sponsored by an agency of the United States government. Neither the United States government nor Lawrence Livermore National Security, LLC, nor any of their employees makes any warranty, expressed or implied, or assumes any legal liability or responsibility for the accuracy, completeness, or usefulness of any information, apparatus, product, or process disclosed, or represents that its use would not infringe privately owned rights. Reference herein to any specific commercial product, process, or service by trade name, trademark, manufacturer, or otherwise does not necessarily constitute or imply its endorsement, recommendation, or favoring by the United States government or Lawrence Livermore National Security, LLC. The views and opinions of authors expressed herein do not necessarily state or reflect those of the United States government or Lawrence Livermore National Security, LLC, and shall not be used for advertising or product endorsement purposes.

This work performed under the auspices of the U.S. Department of Energy by Lawrence Livermore National Laboratory under Contract DE-AC52-07NA27344. 


\title{
Advanced discrete-continuum methods for dynamic loading of tunnels by ground shocks
}

\author{
Oleg Vorobiev
}

Final Report, DTRA Basic Research Award\#10-41581

April 3, 2013 
This work performed under the auspices of the U.S. Department of Energy by Lawrence Livermore National Laboratory under Contract DE-AC5207NA27344 


\section{Contents}

1 Introduction 4

1.1 Review of existing discrete approaches . . . . . . . . . . 4

1.2 Problems with existing equivalent continuum approaches . . . . . 7

1.3 Research goals and objectives . . . . . . . . . . . . 8

1.4 Summary of the current approach . . . . . . . . . . . 8

2 Computational model 13

2.1 Constitutive Modeling framework . . . . . . . . . . . . . 13

2.2 Contact formulation and joint model . . . . . . . . . . . . 22

2.3 Meshing Representative Volumes for jointed rock . . . . . . . . 28

2.3.1 Two and Three joint set model . . . . . . . . . . . 28

2.3 .2 Random joint model . . . . . . . . . . . . . . . . 28

2.4 Meso scale studies for jointed rock masses . . . . . . . . . . . . 29

2.4.1 Scaling the yield surface for jointed rocks . . . . . . . . 29

2.4.2 Poroelasticity of jointed rocks . . . . . . . . . . . 30

3 Discrete-Continuum methods for shock waves in jointed rock masses 36

3.1 Hybrid element solvers . . . . . . . . . . . . . . . . . 36

3.2 Hybrid contact solver . . . . . . . . . . . . . . . . 36

3.2.1 Advection of history variables at the contacts . . . . . . 36

3.2.2 Efficient search algorithm for hybrid contacts . . . . . . 37

3.3 Discrete modeling of fracture . . . . . . . . . . . . . . . 40

3.4 Dynamic element decoupling algorithm . . . . . . . . . . . . 42

3.5 Combinig discrete and continuum methods to model wave propagation in a jointed rock formation . . . . . . . . . . . . 44

3.6 3D application of element decoupling algorithm for dynamic tunnel collapse . . . . . . . . . . . . . . . . . 45

4 Validation and sensitivity study $\quad \mathbf{5 2}$

4.1 Large scale model validations . . . . . . . . . . . . . . . . 52

4.2 Code coupling and discrete-continuum calculations . . . . . . . 53

4.3 Wave focusing and shear motion caused by joints . . . . . . . 54 
5 Conclusion $\quad 61$

5.1 Main achievements of the project . . . . . . . . . . 61

5.2 Benefits to other projects . . . . . . . . . . . 62 


\section{Chapter 1}

\section{Introduction}

It has been long recognized that ignoring discrete nature of rock masses and engineering structures such as tunnel walls in numerical analysis may lead to wrong results $[1,2,3]$. On the other hand, accounting for every single joint in these structures is not computationally feasible. Therefore, for many practical applications using derived homogenized mechanical properties for such systems may be highly desirable. In the case of shock wave loading the structure may experience strong deformations causing blocks to split and slide along the joints. In these situations the homogenized model cannot replace detailed numerical analysis which accounts for the discrete properties of the structure. The present research is an attempt to advance on two fronts. First, is to develop a computational framework to model mechanical systems with multiple discontinuities such as cracks, joints, faults etc which would combine both discrete and continuum methods and transition from one to the other when needed. The second aspect is to apply the methods developed to study the response of such system in relevant engineering applications. The next section will review top of art computational approaches developed by other authors and identify the drawbacks of theses approaches to be improved in the current work.

\subsection{Review of existing discrete approaches}

Discrete structures have been modeled using both Discrete Element (DE) codes and Finite Element (FE) or Finite Difference (FD) codes. In DE codes, for example, walls are typically represented as assemblies of rigid (or linear elastic) blocks with frictional contacts, where the forces acting on the walls may be separately calculated in CFD codes. Some advanced DE codes can model block fracture, bulking and nonlinear normal stiffness at the contacts [4]. FE/FD

codes model each brick as a separate computational domain with simple penalty contacts applied at the boundaries [5]. Advanced continuum models with hardening, softening and nonlinear material response can be used for the blocks. One should mention the following difficulties of the mentioned above discrete 
approaches for shock wave loading simulations: It was found in [6] that depending on the load intensity the jointed media behaves differently. Figures.1.1-1.2 show cylindrical shock wave propagating through jointed rock calculated with GEODYN-L (see details in [6]), Lagrangian parallel FD code with advanced contact algorithm, for two different energy densities and different joint densities. At high pressures, corresponding to a higher energy density, (Fig.1.1) the joints are locked and the response is similar to a continuum response. In the other case (Fig.1.2), when the pressure in the wave is lower the anisotropy of the media becomes obvious. The waves propagate effectively in normal-to-joint directions. The higher the joint density, the stronger is the anisotropy effect. Also, due to finite compressibility of the joints, the wave propagates slower in the presence of joints.

A

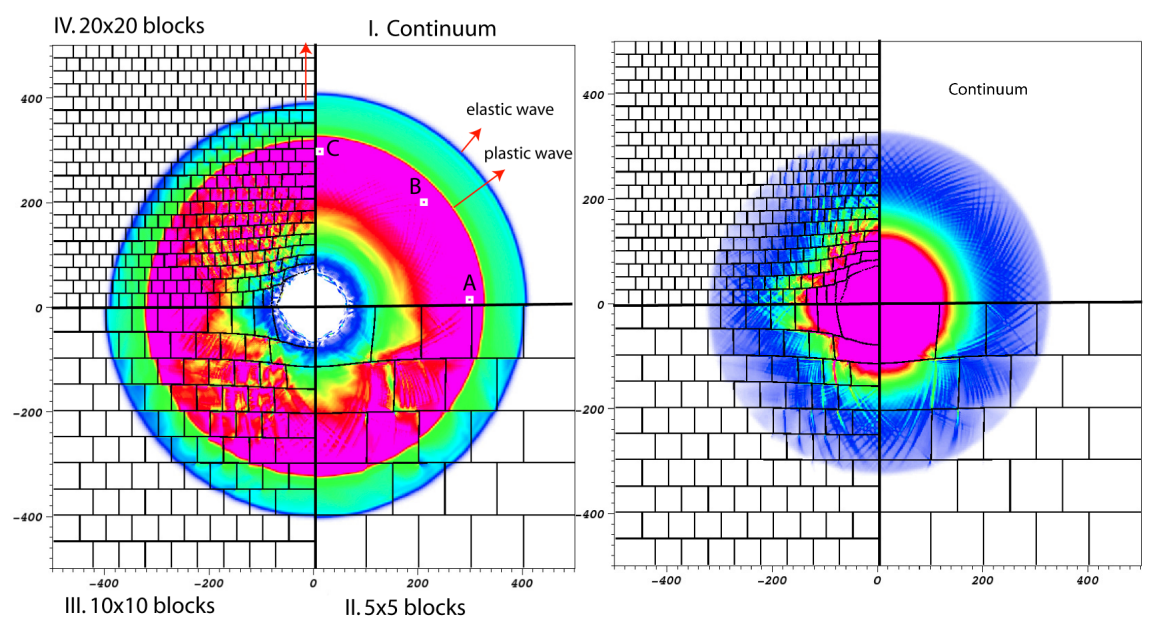

Figure 1.1: Pressure (A) and plastic strain (B)contours at time $100 \mu s$ for different joint densities. The pressure range is $0-0.2 \mathrm{GPa}$ and the strain range is $0-0.1$. The specific energy for the source is $50 \mathrm{~kJ} / \mathrm{g}$ and the friction angle used for the joints is 21 degree.

For waves propagating in a heavily jointed medium, contacts are stiff only if cracks and joints are strongly confined, so that the deformations are mainly absorbed by the medium. In this case the joints can be considered as incompressible slide boundaries. When the waves propagate away from the energy source, they attenuate, confining the joints less and less. At large distances the joint response is linear and its elastic compliance is typically much high that of the solid. In this situation the joints cannot be modeled as incompressible boundaries. The impedance mismatch causes the wave to reflect back from the joints. Multiple reflections between joints increase the path for the wave and cause enhanced attenuation, especially if the wave length is less or comparable to the joint spacing. Thus, development of an efficient numerical technique 
A

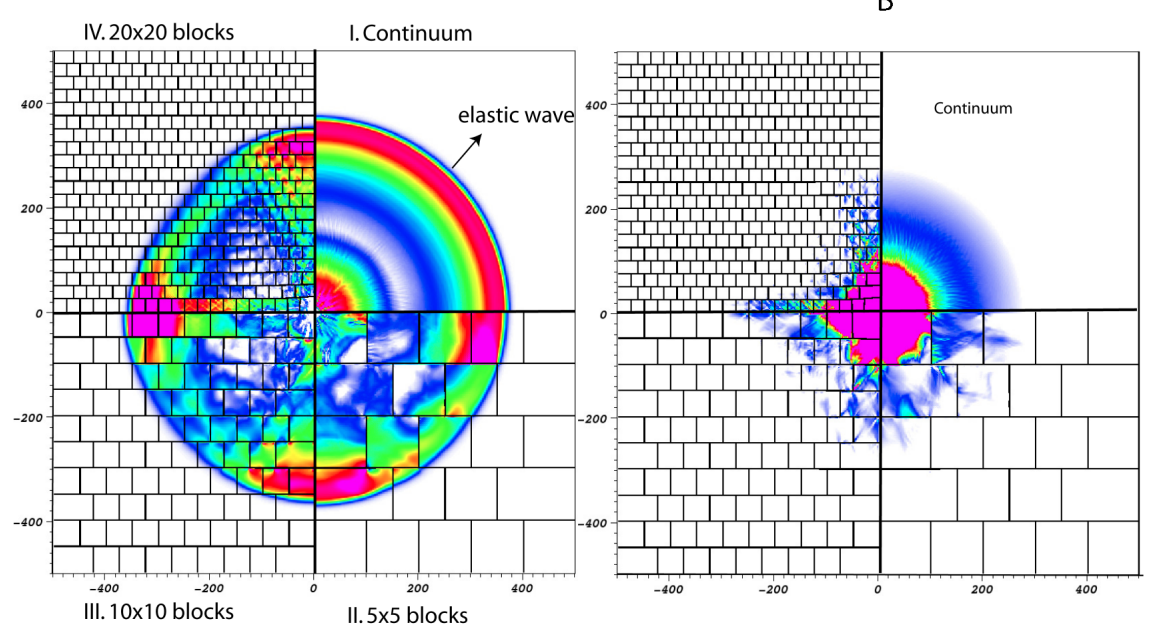

Figure 1.2: Pressure (A) and plastic strain (B)contours at time $100 \mu \mathrm{s}$ for different joint densities. The pressure range is $0-0.05 \mathrm{GPa}$ and the strain range is $0-0.01$. The specific energy for the source is $5 \mathrm{~kJ} / \mathrm{g}$ and the friction angle used for the joints is 11 degree.

which would treat both complaint and incompressible joints is very important for the applications considered.

To summarize one can list the following limitations of these approaches:

- At high pressures the joints become locked, and their normal stiffness becomes few orders of magnitude higher. Most of DE codes are not designed to model shock wave propagation through the media with stiff joints. Small time step dictated by stability conditions in the explicit algorithm makes them prohibitively expensive for such problems.

- Most of DE codes apply simplistic material models for the blocks (linear elasticity or even rigid blocks) which can give wrong results if the blocks are subject to large distortions and pressures.

- Most of FE/FD codes use non-compliant contacts which is only appropriate at high pressures when the joints are locked. Thus, the presence of such contacts does not have any effect on the wave propagation speed and their attenuation.

- Penalty contacts often used by FE/FD/DE codes do not use any history variables and, therefore, cannot apply realistic joints models to describe effects of dilatancy, softening and hardening as well as tensile failure $[7,8]$. Some DE methods may use history variables [4], but these histories seize to exist once the contacts between the blocks disappear. Therefore, they cannot properly describe surface degradation for a block that slides past 
few other blocks, since there is no history variable advection between the neighboring contact elements.

- Many FD/FE codes are not designed to handle multiple contacts which have been traditionally the area of application for DE methods. Development of robust contact algorithms for parallel machines to model heavily jointed media is important for the applications considered.

In the current work limitations mentioned above will be addressed.

\subsection{Problems with existing equivalent contin- uum approaches}

Because of computational difficulties in explicit modeling of the structures with multiple joints the main focus of the research in that area is on the development of homogenization failure criteria for the discrete engineering structures $[9,10,3]$. Homogenized continuum models are then used in the FE codes to predict available experimental data. A linear elastic transversely isotropic Cauchy material is often assumed for the medium with the frictional sliding along the joints controlling the shear stress. Some researches $[3,2]$ have found that nonorthotropic continuum models are needed to better describe experimental data especially when material fails.

The existing continuum approaches have the following limitations:

- Since most of continuum models for masonry are linear elastic they cannot be applied to the shock wave propagation through the walls. Therefore, the range of their application is restricted to the analysis of the wall damage due to an air-blast.

- Continuum methods with material softening suffer mesh sensitivities.

- Continuum methods, applied in Lagrangian FE/FD codes, do not model separation of the bricks and their fragments mainly because of the mesh tangling.

Tunnel stability in hard rock under dynamic shock loading conditions is not well understood. Numerical simulation of such problems is very challenging and requires many steps before this mission can be accomplished. These steps include:

- Constitutive modeling of rock samples describing porous compaction, bulking, spall, distortional damage and failure taking place during shock loading,

- Methods to upscale physical properties from small rock sample to rock masses in order to use continuum approaches in large scale modeling, 
- Advanced numerical techniques to resolve thin structures such as cracks, joints and faults, where it is necessary, as well as the constitutive relations for these discrete structures,

- Advanced numerical algorithms for large-scale parallel computing and availability of computer clusters, where such analysis can be performed

As it will be shown in this report, the developed tools and methodology can help us to answer important questions such as:

- How shock waves propagate and attenuate in a jointed rock mass?

- How anisotropy in wave attenuation due to the joint distribution in the rock effects the deformation and failure of the tunnel?

- How the modes of failure in the tunnel walls depend on various factors (depth of the tunnel, quality of the rock mass, direction and intensity of the loading etc)?

- How to build better continuum model for jointed rock for dynamic conditions?

Answering the last questions is important, since in practical applications

continuum approaches remain the main work horse to perform evaluation of tunnel stability.

\subsection{Research goals and objectives}

The purpose of this research was to develop effective numerical tools and methodology to model shock wave propagation through discrete engineering structures such as tunnel walls and randomly jointed rock masses. These tools will be applied in the future to the analysis of tunnel failure under the shock wave loading.

\subsection{Summary of the current approach}

A strongly nonlinear joint model developed for GEODYN-L code [11] is applied both to shock waves and linear waves propagating through the jointed rock. Implicit integration of constitutive equations for joints described in [11] helps avoiding time step limitations suffered by explicit integration schemes. Simple Common Plane contact algorithm, designed and implemented for parallel computing with distributed memory, is used to solve large scale-problems, where the maximum allowable size of the problem is limited only by the number of CPUs used in calculations.

The method outlined in [12] is used to build better continuum models for jointed rock masses, where representative volume is loaded triaxially in numerical experiment to study the equivalent response for the medium. 
Continuum representation of jointed structures is used in big scale practical calculations together with the discrete ones. Discrete methods can be applied only in the areas where the discrete nature of the structural response is very important, for example, in the close proximity to the tunnel wall.

The material model used for rock blocks describes mechanical response observed in rock materials under triaxial and uniaxial compression. Figure 1.3 illustrates how this model describes measured compaction curves for the limestone samples of various porosity. The model captures effects of shear-enhanced compaction as it is shown in Fig.1.4 where uniaxial and hydrostatic compaction of sandstones of different porosity is compared with the model.

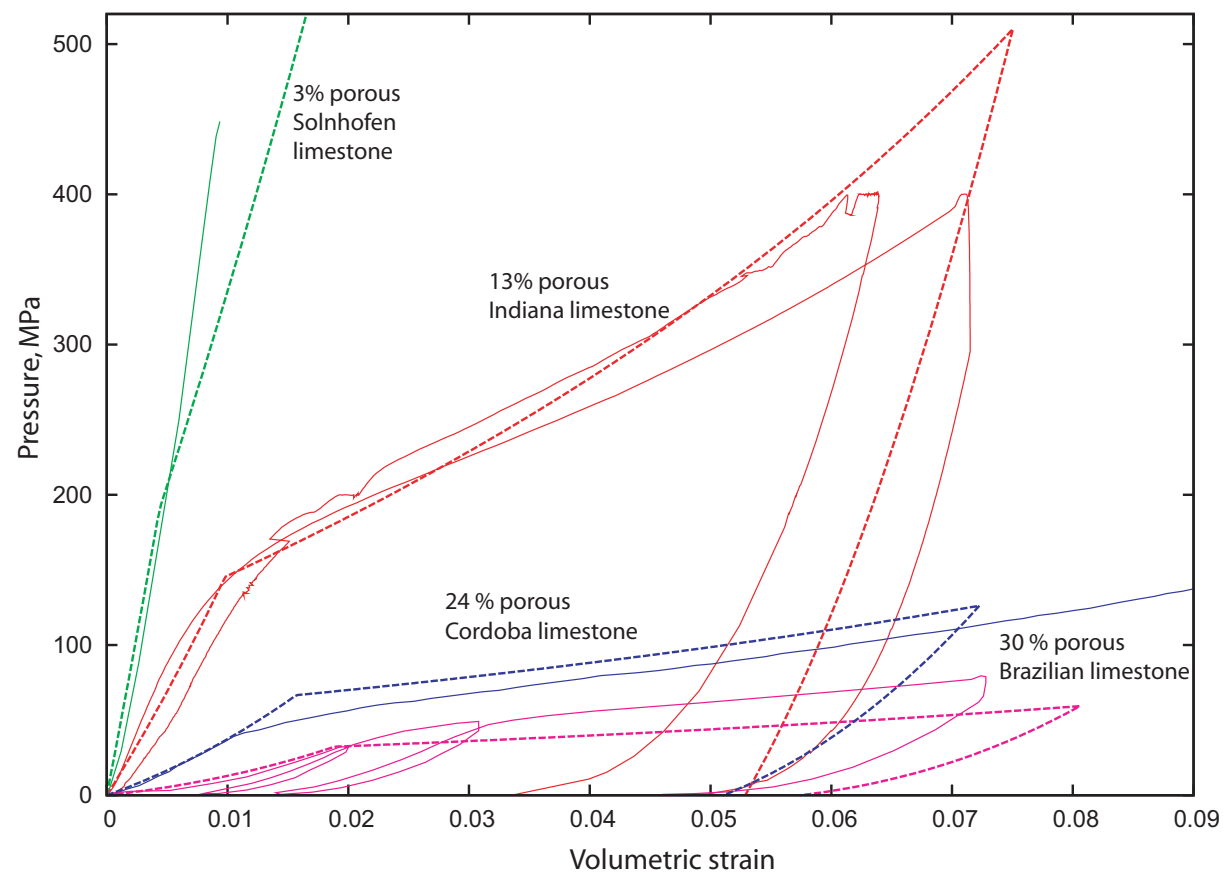

Figure 1.3: Comparison of experimental and calculated hydrostatic compaction curves for limestones with different reference porosities. Solnhofen limestone data are from [13], Cordoba Limestone data are from [14], Brazilian limestone data are from [15], Indiana limestone data are the same as in [16]

The material model used in this study is thermodynamically consistent and applicable to shock wave loading regime. It was developed to be applied not only for small scale rock samples but also for isotropic rock masses under assumptions that the joints are randomly distributed and overall response remains isotropic.

To derive parameters for continuum model describing the rock mass, a computational model for a representative volume (RV) is built as an assembly of subdiscretized rock blocks interacting through the joints. This model is verified and validated at different levels. Triaxial test data are used to validate material 


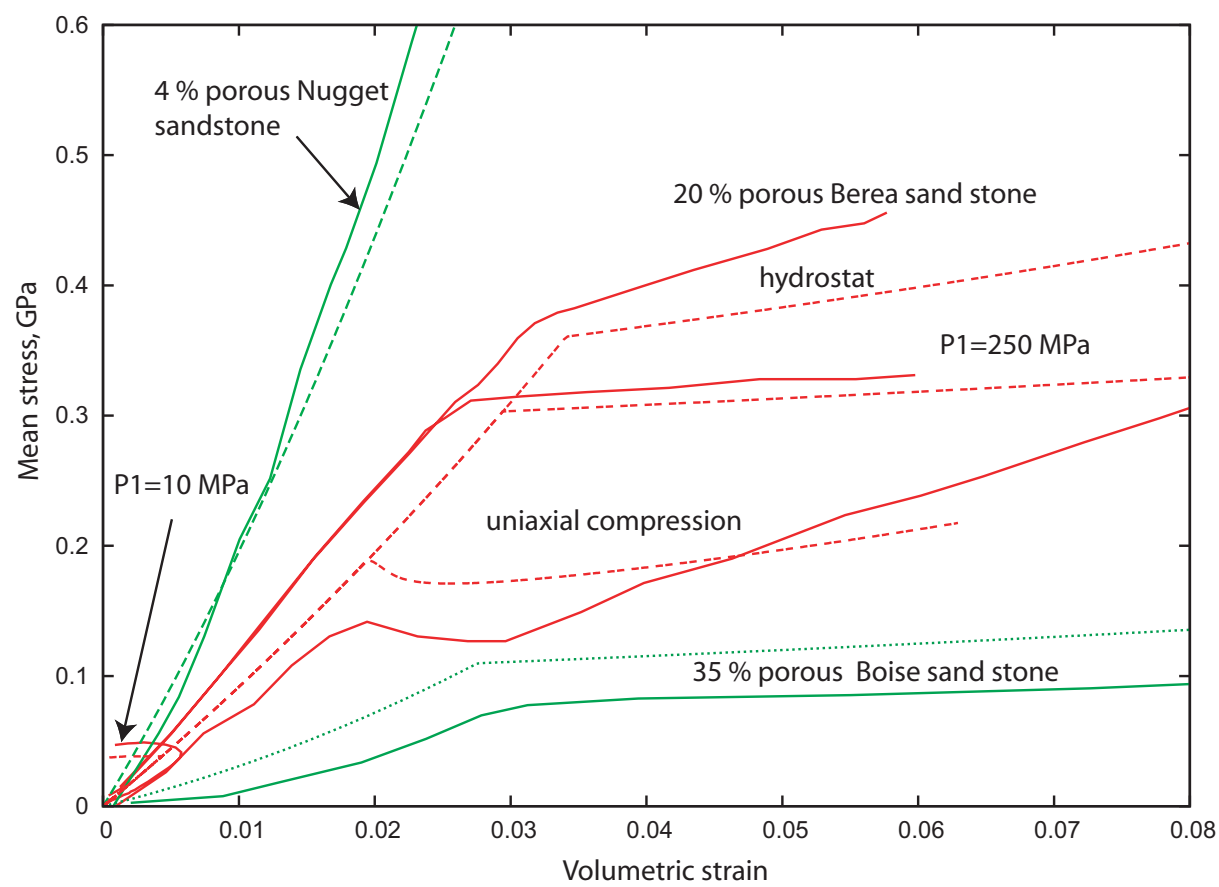

Figure 1.4: Comparison of experimental (solid) and calculated (dashed) compaction curves for sandstones with different reference porosities. Boise sandstone data are from [17], Berea sandstone data are from [13], Nugget sandstone data are from $[18,19]$

response at the block level. Shear tests performed for the joints are used to validate the joint model. Verification tests are performed to show that the joints model is implemented properly in RV. Finally, the field scale calculations are performed to validate the model for available series of underground explosions.

The model has been tested in simulations of the ground motion due to underground explosions in limestones. Three different experiments on spherical wave generation in limestone were simulated with the present model. The first one is a lab-scale experiment with $12 \%$ porous Salem limestone described in [16]. The second one is an underground test described in [20]. And, the last one is a series of HE cavity decoupling tests in limestone performed in Kirghizia in 1960 $([21])$. The limestones encountered in these tests have different porosities $(12 \%$, $2 \%$ and $0.5 \%$ ), therefore modeling the three tests with a single set of parameters presents significant challenge. The same set of parameters was used in the calculations as the one used to model the triaxial test for various limestones. The peak velocities and displacements calculated for the tests at different ranges are shown in Fig.1.5. Experimental set-ups for the Salem limestone explosion and some symmetric explosions in Kirghizian caves (where the charge was located in the middle of the cave) allowed calculations to be performed for the $1 \mathrm{D}$ case. 
Test II was performed in an underground chamber of rectangular shape, which makes it a 3D problem. Ma [20] approximated the chamber by a cylinder of equivalent volume to reduce analysis to a $2 \mathrm{D}$ case. Similar problem set-up was used in the present calculation as well as a more simple 1D calculation, where the chamber was approximated by a $2 \mathrm{~m}$ sphere. The difference between the $1 \mathrm{D}$ and the 2D velocity profiles at the $8 \mathrm{~m}$ gauge reported in [20] can be seen in the left low corner of Fig.1.5. Calculation with the intact model for the same problem is shown with the dashed line, the experimental record is shown with the thick solid line, 1D calculation with the in situ model is shown with the dotted line and the $2 \mathrm{D}$ result is shown with the thin solid line. The second hump in the profile, according to the calculations, is attributed to the reflection of the blast wave from the radial walls of the chamber. From the peak velocity attenuation it is seen that $2 \mathrm{D}$ calculations (the thick line, Test II) give better agreement with the experimental data (shown with the squares). This is because less of the energy of the HE is coupled to the rock for the cylindrical chamber. Also, intact model does not seem to provide the right shape of the pulse underestimating both velocity and displacement.

Test III includes an explosion in a $2.88 \mathrm{~m}$ radius spherical cavity for both decoupled and tamped cases. The density of limestone was around $2.7 \mathrm{~g} / \mathrm{cm} 3$ and the P-wave velocity was between 5.5 and $6 \mathrm{~km} / \mathrm{s}$.

Results of decoupled test simulation showed reasonable agreement with experimental data (shown with crosses). Using in situ model with extra $0.5 \%$ of joint porosity (thick lines) gives better agreement with the experiment compared to the intact model (thin lines). Simulations performed by Murphy [21] showed significantly overestimated peak velocities. As far as the tamped test is concerned, the calculations seem to underestimate both the peak velocity and displacement especially at the long ranges. Better agreement with the experiment (shown with diamonds) can be obtained if some additional porosity is added to the limestone surrounding the charge. Calculated peak velocity attenuation curves for three different porosities $(0.5 \%, 11 \%, 21 \%)$ for the limestone around the charge are shown in Fig.1.5.

The model has been also validated for granite. Both intact and inSitu granite models were developed and used to be applied in calculations withing Source Physics Experiment Program (SPE). Results of this validation are presented in the report.

The performed parametric studies using advanced numerical techniques for wave propagation in continuous and discontinuous media increase understanding of the processes involved in actual wall collapse and tunnel failure under dynamic loading. The developed effective numerical tools and methods will dramatically assist and benefit the current counter-WMD mission. 


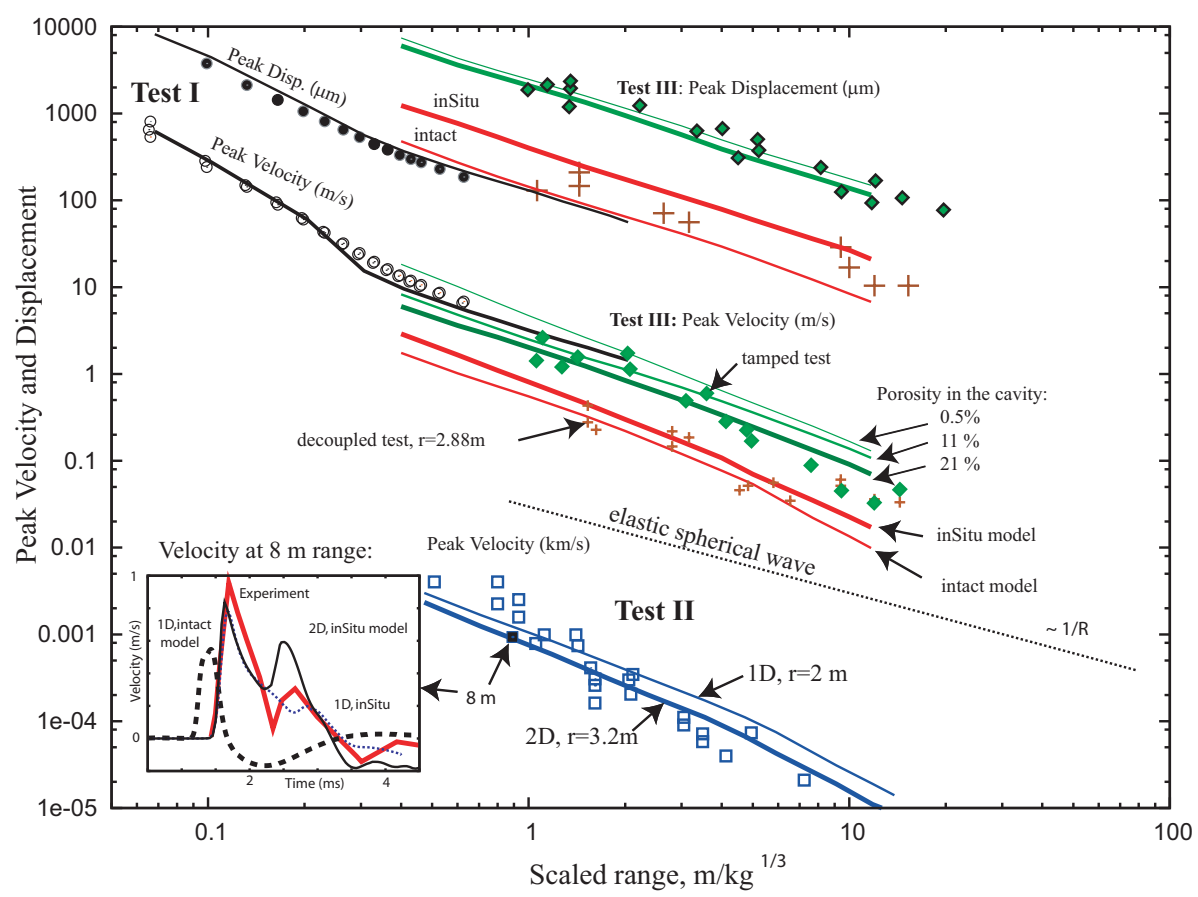

Figure 1.5: Comparison of simulated (solid lines) and observed (markers) peak velocities and peak displacements for various test in limestone. 


\section{Chapter 2}

\section{Computational model}

\subsection{Constitutive Modeling framework}

The thermomechanical structure of the model is based on the developments in $[22,23,12]$. Within this context, an elemental volume $d v$ of the porous material in the present configuration expresses as the sum of solid volume and pore volume, such that

$$
d v=d v_{s}+d v_{p}, \quad d V=d V_{s}+d V_{p},
$$

where $\left\{d V, d V_{s}, d V_{p}\right\}$ are the values of $\left\{d v, d v_{s}, d v_{p}\right\}$, respectively, in a fixed reference configuration. The porosity $\phi$ and its reference value $\Phi$ are defined by

$$
\phi=\frac{d v_{p}}{d v}, \quad \Phi=\frac{d V_{p}}{d V}
$$

The total dilatation, $J$, and the average dilatation of the solid, $J_{s}$, are defined by

$$
J=\frac{d v}{d V}, \quad J_{s}=\frac{d v_{s}}{d V_{s}}=\left(\frac{1-\phi}{1-\Phi}\right) J
$$

The total dilatation, $J$, is determined by the evolution equation

$$
\frac{\dot{J}}{J}=D \bullet I, \quad D=\frac{1}{2}\left(L+L^{T}\right)
$$

where $\mathbf{L}$ is the velocity gradient tensor.

A symmetric unimodular tensor $B_{e^{\prime}}$ is used as a measure of pure elastic distortion in the evolution equation

$$
\dot{B}_{e}^{\prime}=L B_{e}^{\prime}+B_{e}^{\prime} L^{T}-\frac{2}{3}(D \bullet I) B_{e}^{\prime}-A_{p}, A_{p}=\Gamma_{p}\left(B_{e}^{\prime}-\left(\frac{3}{B_{e}^{\prime-1} \bullet I}\right)\right),
$$

where the tensor, $A_{p}$, characterizes the direction and magnitude of inelasticity for distortional response and $\Gamma_{p}$ requires an additional constitutive equation (see [23]). 
In contrast to many other purely mechanical models used for rocks and geologic materials $[24,25]$ the current model is a thermo-mechanical one. The Helmholtz free energy $\Psi$ is chosen as a function of the variables $J_{s}$, an invariant $\alpha_{1}=B_{e}^{\prime} \bullet I$ of $B_{e^{\prime}}$ and temperature $\Theta$ :

$$
\begin{aligned}
& \Psi\left(J_{s}, \Theta, \alpha_{1}\right)=\hat{\Psi}\left(J_{s}, \Theta\right)+\frac{1}{2} G\left(J_{s}, \Theta\right)\left(\alpha_{1}-3\right) / \rho_{s 0} \\
& \hat{\Psi}\left(J_{s}, \Theta\right)=\varepsilon_{c}\left(J_{s}\right)+\Psi_{T}\left(J_{s}, \Theta\right)
\end{aligned}
$$

If we neglect small terms in pressure related to $\alpha_{1}$ then the stress tensor $T$ can be expressed as:

$$
\begin{aligned}
& T=-p I+T^{\prime}, p=-\rho_{0} \frac{\partial \Psi}{\partial J}=\left(1-\phi-J \frac{\partial \phi}{\partial J}\right) p_{s} \\
& p_{s}\left(J_{s}, \Theta\right)=-\rho_{s 0} \frac{\partial \Psi}{\partial J_{s}}, \quad T_{s^{\prime}}=J_{s}^{-1} G\left(J_{s}, \Theta\right) B_{e^{\prime \prime}}
\end{aligned}
$$

where $p$ is the pressure, $T^{\prime}$ is the deviatoric part of the stress, $B_{e}^{\prime \prime}$ is the deviatoric part of $B_{e}^{\prime}$ and $p_{s}$ and $T_{s^{\prime}}$ are the pressure and deviatoric stress of the solid matrix, respectively. In shock wave physics the part of the Helmholtz free energy $\hat{\Psi}\left(J_{s}, \Theta\right)$ is often expressed as a sum of volume-dependent potential $\varepsilon_{c}\left(J_{s}\right)$ (the cold part) and the thermal part representing anharmonicity and electronic contribution at high temperatures $\Psi_{T}\left(J_{s}, \Theta\right)$. The solid pressure, $p_{s}$, is then found as the derivative of the free energy with respect to volume shown in Eq (2.7). Thus, the solid pressure consists of two parts (the cold part and the thermal part). The thermal pressure is convenient to express in terms of specific internal energy, $\varepsilon$, instead of the temperature. One of the simplest of this type, the Mie-Gruneisen EOS, can be written as

$$
\begin{aligned}
& p_{s}=p_{c}\left(J_{s}\right)+\Gamma \rho_{s 0}\left(\varepsilon-\varepsilon_{c}\left(J_{s}\right)\right) \\
& p_{c}\left(J_{s}\right)=-\rho_{s 0} \frac{\partial \varepsilon_{c}\left(J_{s}\right)}{\partial J_{s}}
\end{aligned}
$$

The reference curve $p_{c}\left(J_{s}\right)$ can be derived from shock experiments or approximations of the potential $\varepsilon_{c}\left(J_{s}\right)$. The thermal pressure part is proportional to the specific energy $\varepsilon$, with proportionality coefficient $\Gamma$ called the Gruneisen coefficient.

Elastic properties and poroelasticity Experimental data on hydrostatic compression of porous rocks show nonlinear elastic response up to 10-100 $\mathrm{MPa}$, which can be attributed to the elastic closing of the microcracks [27]. For a jointed rock medium poroelasticity can be enhanced due to the presence of joints in addition to the microcracks. We will not separate joint porosity from the incipient porosity in the rock blocks. Yet, some poroelasticity parameters can be made explicitly dependent on the joint density. To model nonlinear elastic response porosity is expressed as a function of $J$ and a history dependent unloaded porosity $\phi_{u}$ as

$$
\phi=\frac{\phi_{u}}{(1+x)}, x=\frac{a\left(1-\phi_{u}\right)\left(J_{u} / J-1\right)}{\phi_{u}}, J_{u}=\frac{1-\Phi}{1-\phi_{u}}
$$




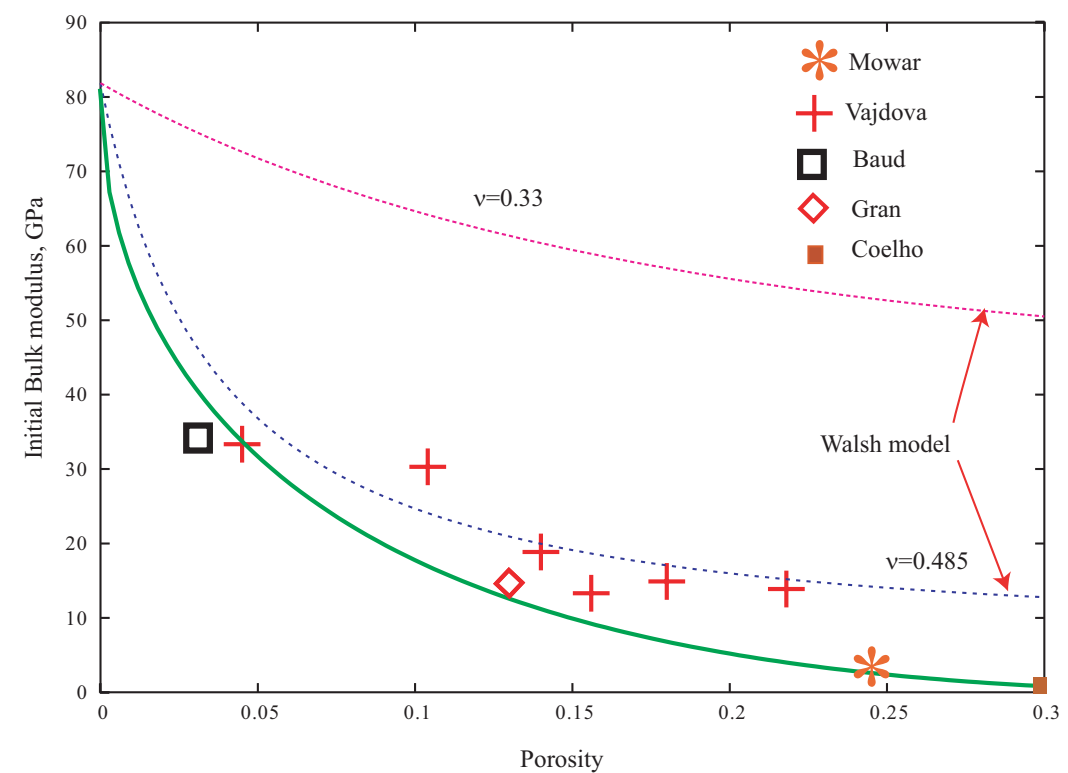

Figure 2.1: Porosity - initial bulk modulus correlation for various limestones. The solid line is the results for the current model, the dashed lines are predictions with Walsh model [26] using two different Poisson ratios, the markers are the experimental data.

The unloaded porosity $\phi_{u}$ is the porosity that would exist if the material was unloaded from the current state. This formulation provides the correct asymptotic behavior and can be shown to satisfy the second law of thermodynamics if coupled with the evolution equations for $\phi_{u}$ described later. Selection of the functional form for Eq. (2.9) was motivated by the work of [22]. The advantage of Eq. (2.9) is that it provides an analytic expression for unloaded porosity at given porosity and compression.

Using Eq. (2.7) and Eq. (2.9) the bulk modulus, $K$, can be written as

$$
K=-J \frac{\partial p}{\partial J}=K_{s}\left(1-\phi_{u}\right)\left(1-\frac{a J_{u}}{J(1+x)^{2}}\right)^{2}-\frac{2 a^{2}\left(1-\phi_{u}\right)^{2} J_{u}^{2}}{J^{2} \phi_{u}(1+x)^{3}} p_{s}
$$

In the unloaded state, where $p_{s} \rightarrow 0, x \rightarrow 0, J_{u} / J \rightarrow 1$, the initial bulk modulus $K_{0}$ is equal to a fraction of the solid modulus $K_{s}$ :

$$
K_{0} \rightarrow K_{s}(1-\Phi)(1-a(\Phi))^{2}, \quad K_{s}=-J_{s} \frac{\partial P_{s}}{\partial J_{s}}
$$

By choosing an appropriate function for $a\left(\Phi, s_{j}\right)$ one can adequately describe the observed initial bulk modulus dependence on porosity, $\Phi$, and the joint density, $s_{j}$. Fig. 2.1 shows a comparison of the bulk modulus calculated using Eq. (2.11) versus experimental data for limestones of various porosity. 
Bulk modulus reduction with porosity can be explained with a simple model [26] considering an elastic matrix embedded with spherical pores. According to that model, the effective compressibility of the rock is expressed as

$$
\frac{1}{K_{0}}=\frac{1}{K_{s}}\left(1+\frac{3(1-\nu) \Phi}{2(1-2 \nu)(1-\Phi)}\right)
$$

where $\nu$ is the Poisson ratio. As shown in Fig. 2.1, Eq. (2.12) requires unrealistically high values of the Poisson ratio in order to match experimental data.

Porous compaction and dilation A linear law, similar to one described in [28], is used to reduce the unloaded porosity, $\phi_{u}$, during compaction. For simplicity, strain rate effects are not considered in the present paper. The unloaded porosity is constrained by the compaction curve as

$$
\phi_{u} \leq \phi_{u}^{\max }=\Phi-S(\Phi)(1-\Phi)\left\langle\mu+\mu_{e}+\mu_{s}-\mu_{b}-\mu_{c}(\Phi)\right\rangle,
$$

where $\mu=1 / J-1$, and $\mu_{c}$ is the volumetric strain corresponding to the onset of compaction.

After Eq. (2.13) is applied the new value of porosity, $\phi$, is found from Eq. (2.9) and the pressure is recalculated by Eq. (2.7).

The shifts $\mu_{b}, \mu_{s}, \mu_{e}$ model effects of bulking, shear enhanced compaction and thermal softening. The thermal shift, $\mu_{e}$, is found from the linearized MieGruneisen EOS assuming that compaction will begin at $P \geq P_{c 0}$ even if material is heated at constant volume.

$$
\begin{aligned}
& P=(1-\Phi) P_{s} \approx(1-\Phi) K_{s}\left(\mu+\mu_{e}\right) \\
& P_{c 0} \approx(1-\Phi) K_{s} \mu_{c}(\Phi) \\
& \mu_{e}=\Gamma \varepsilon /\left((1-\Phi) \partial p_{s} / \partial \rho_{s}\right)
\end{aligned}
$$

This shift accounts for enhanced compaction due to thermal pressure.

The bulking shift, $\mu_{b}$, is proportional to the amount of bulking porosity, $\phi_{2}$, as

$$
\mu_{b}=\frac{\bar{\gamma} \phi_{2}}{S(\Phi)(1-\Phi)}
$$

where $\bar{\gamma}$ controls the rate of compaction of porosity produced by bulking.

The bulking porosity, $\phi_{2}$, is a history variable describing extra porosity produced by dilatancy using the following equation:

$$
\dot{\phi}_{2}=\frac{A_{0}+A_{1}\langle d Y / d p\rangle}{1+\langle d Y / d p\rangle}\langle d Y / d p\rangle \dot{\varepsilon}_{p}\left(1-\phi_{u}\right)
$$

Because the rate of bulking is proportional to the positive slope of the yield surface, dilatancy and brittle material response will only take place at low confinements where the cap is not applied to the yield surface. The parameters $A_{0}$ and $A_{1}$ control the degree of associativity. 
The shear-enhanced compaction shift found from the assumption that yielding and compaction take place simultaneously (see more details in [28]) is expressed as

$$
\mu_{s}=\frac{P_{c}(\Phi)}{K}(1-\xi)\left(\frac{\sigma_{e}}{C Y_{f}(p)}\right)^{1 / r},
$$

where $\sigma_{e}$ is the current Von Mises Stress, $K$, is the current bulk modulus and $P_{c}$ is the current compaction pressure. The failure surface, $Y_{f}(p)$, is described in the following section. The compaction pressure, $P_{c}$, is found as

$$
P_{c}(\Phi)=p+K\left(\frac{\Phi-\phi_{u}}{S(\Phi)\left(1-\phi_{u}\right)}+\mu_{c}(\Phi)+\mu_{e}+\mu_{b}-1 / J\right)
$$

The rate of compaction is defined by the slope, $S(\Phi)$, where $0 \leq S(\Phi) \leq 1$. The smaller the slope, $\mathrm{S}$, the slower is the compaction. For $\mathrm{S}=1$, compaction takes place at a constant pressure.

Compaction starts earlier when deviatoric stress is present (shear-enhanced compaction effect). The compaction slope is reduced in the presence of deviatoric stresses (when $\mu_{s} \geq 0$ ), as

$$
S(\Phi)=\frac{\Phi S_{0}(\Phi)}{\Phi+\mu_{s} S_{0}(\Phi)(1-\Phi)},
$$

where $S_{0}(\Phi)$ is the compaction slope for the hydrostatic condition (when $\mu_{s}=$ 0 )defined as a function of the reference porosity, $\Phi$. Eq. (2.19) is derived from the assumption that at full compaction, when $\phi_{u} \rightarrow 0$, the material will have the same density regardless of the loading path.

The function $S_{0}(\Phi)$ can be found by modeling hydrostatic compression of intact rocks with different porosity. Fig. 1.3 and Fig. 1.4 show hydrostatic compaction curves for limestones and sandstones of various porosity. The dashed lines are calculations with the current model, the solid lines are experimental data. The experimental data show that, for high porosity rocks, compaction starts at lower pressures and is more pronounced. The initial value of the crush pressure, $P_{c 0}$, depends on the reference porosity as well as on the grain size. The Hertzian fracture model relates the onset of the grain crushing with the porosity as

$$
P_{c 0}=2.2 \frac{\left(1-\nu^{2}\right)^{2} K_{I C}^{3}}{E^{2}(1-2 \nu)^{3}(\Phi c)^{3 / 2}},
$$

where $c$ is the length of any pre-existing crack and $K_{I C}$ is the fracture toughness coefficient. It is reasonable to assume that the crack length, $c$, is proportional to the grain size. This argument is often used to explain the observed dependence of $P_{c 0}$ on the grain size and porosity for limestones [27] and sandstones [29].

Information on grain size is not easily available, but very often low-porosity samples have smaller grain size than those that are more porous. Therefore, it is reasonable to assume that $P_{c 0}$ depends mainly on porosity. It is interesting to note that since both $P_{c 0}$ and the initial bulk modulus, $K_{0}$, decrease with porosity, the strain to compaction, $\mu_{c}$, defined as $\mu_{c}=\frac{P_{c 0}}{K_{0}}$, is less sensitive to 
porosity. For example, for limestones with $7.5-15 \%$ porosity $\mu_{c}=0.01 \pm 0.001$. The model captures the effect of shear-enhanced compaction observed (for example) in Berea sandstone (see Fig. 1.4). For uniaxial compression the porous compaction begins at lower volumetric strains. Two cases of triaxial compression, corresponding to confinement pressures of $\mathrm{P} 1=10 \mathrm{MPa}$ and $\mathrm{P} 1=250 \mathrm{MPa}$, are also shown in Fig. 1.4. The effect of bulking (dilatancy) is captured by the model for the low confinement case $(\mathrm{P} 1=10 \mathrm{MPa})$.

Yield Surface The current model introduces three pressure dependent surfaces that govern the material response during yielding: the initial yield surface (onset of yield), $Y_{0}(p)$, the failure surface, $Y_{f}(p)$, and the residual surface, $Y_{r}(p)$ ( see Fig.2.2).

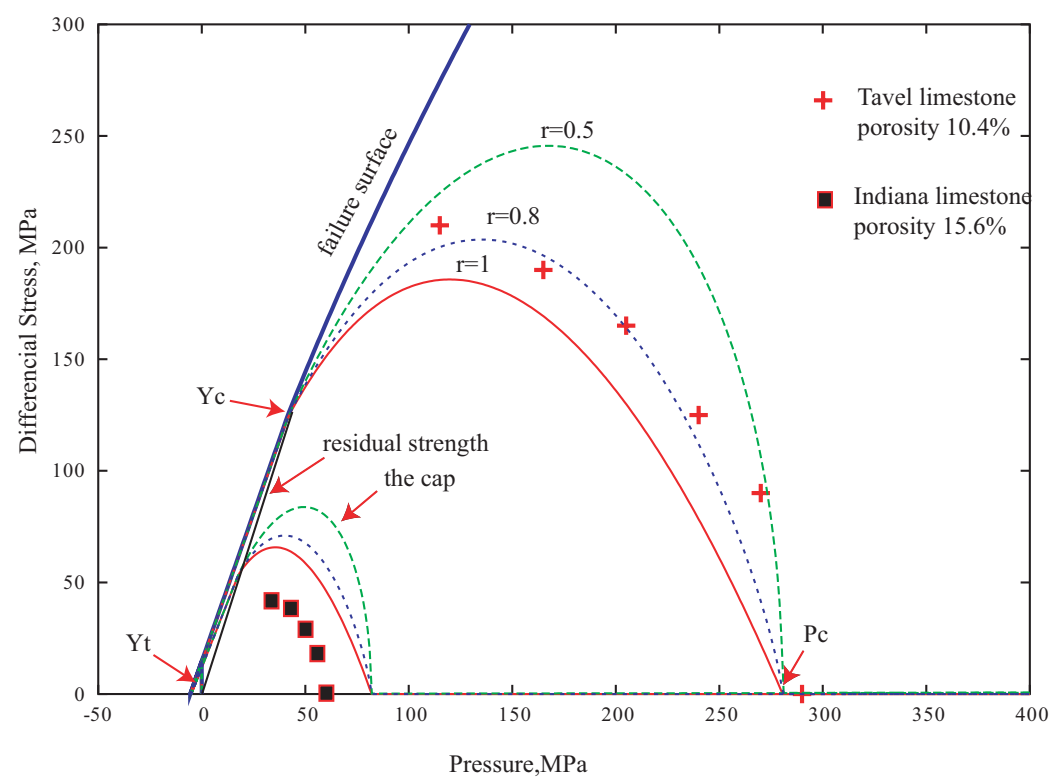

Figure 2.2: Yield surfaces in Y-P plane. The cap is calculated for three different values of $\mathrm{r}$ parameter. The pressure corresponding to the beginning of compaction in hydrostatic conditions $P_{c}(\Phi)$ is defined by the compaction curve

The yield strength corresponding to a generalized triaxial compression state, $Y_{T X C}$, is derived from $Y_{0}, Y_{f}$, and $Y_{r}$ such that

$$
Y_{T X C}\left(p, \delta_{h}, \Omega\right)=\left(\delta_{h} Y_{f}(p)+\left(1-\delta_{h}\right) Y_{0}(p)\right)(1-\Omega)+\Omega Y_{r}(p)
$$

The equivalent plastic strain,$\varepsilon_{p}$, determined by integrating the following 
evolution equation

$$
\dot{\varepsilon}_{p}=\left(\frac{2}{3} D_{p} \bullet D_{p}\right)^{1 / 2}, D_{p}=\frac{1}{2} \Gamma_{p} A_{p} \approx \frac{1}{2} \Gamma_{p} B_{e^{\prime \prime}},
$$

is used to define a hardening parameter $\delta_{h}$ as

$$
\delta_{h}=\frac{\varepsilon_{p}}{\varepsilon_{p}+\varepsilon_{\text {hard }}},
$$

where $\varepsilon_{\text {hard }}$ is a material constant.

The damage, $\Omega$, is assumed to be related to the total amount of bulking porosity (dilatancy) generated in the material, as

$$
\Omega=\frac{\left\langle\phi_{2}-\phi_{c r}\right\rangle D}{1+\left\langle\phi_{2}-\phi_{c r}\right\rangle D}, 0 \leq \Omega \leq 1,
$$

where $\mathrm{D}$ is the rate of softening, and $\phi_{c r}$ is a threshold value of porosity. As damage accumulates during loading, the material softens and its strength approaches the residual curve

$$
Y_{r}(p)=\min \left\{Y_{0}(p), Y_{0}\left(P_{B D}\right) \frac{p}{P_{B D}}\right\},
$$

where $P_{B D}$ is the brittle-ductile transition pressure.

The initial yield surface is expressed in the form

$$
Y_{0}(p)=C Y_{f}(p)\left[\frac{1-p / P_{c}}{1-\xi(\Phi)}\right]^{r}, 0 \leq C \leq 1,
$$

where $C$ and $r$ are material constants. The compaction pressure, $P_{c}(\Phi, J)$, calculated by Eq. (2.18), changes as porosity is compacted. The model parameter $r$ controls the shape of the cap. Fig. 4 shows three initial yield surfaces calculated with $\mathrm{r}=0.5, \mathrm{r}=0.8$ and $\mathrm{r}=1$ as well as experimental initial yield points for Indiana and Tavel limestones. The values $r \leq 0.5$ give the cap with an infinite slope at $P=P_{c 0}$, where $P_{c 0}$ is the pressure corresponding to the onset of compaction.

The function $\xi(\Phi)$ is defined as

$$
\xi(\Phi)=\frac{P_{B D}}{P_{c 0}}=\frac{Y_{c}(\Phi) R_{B D}(\Phi)}{\mu_{c}(\Phi) K_{0}(\Phi)},
$$

where $R_{B D}(\Phi)$ is the ratio of the brittle-ductile transition pressure to the unconfined compressive strength. If no data are available, $R_{B D}$ can be estimated from the intersection of the Mogi line $([30,31])$ with the onset curve $C Y_{f}(p)$.

The ultimate strength function, $Y_{f}(p)$, is based on the H\&B strength criterion $[32,33]$ that relates the maximum $\left(\sigma_{1}\right)$ and minimum $\left(\sigma_{3}\right)$ principal stress on the failure surface as

$$
\sigma_{1}=\sigma_{3}+Y_{c}\left(m \frac{\sigma_{3}}{Y_{c}}+s\right)^{n}
$$


For most rocks $\frac{1}{2}$ is a reasonable value for $n$. The parameter $s$ is equal to unity for intact material and less than unity for in situ material. Hoek in [32] gives an empirical relationship between the coefficientss and $m$ and the Geologic Strength Index (GSI)

$$
s=\exp \left(\frac{G S I-100}{9}\right), m=m_{i} \exp \left(\frac{G S I-100}{28}\right)
$$

In Eq. (2.29), $m_{i}$ is the value of $m$ for intact rock; it can be obtained from static lab tests. For triaxial compression with $\sigma_{e}=Y_{f}$, the principal stresses $\sigma_{1}$ and $\sigma_{3}$ are given by

$$
\sigma_{1}-\sigma_{3}=Y_{f}, \quad \sigma_{3}=p-\frac{Y_{f}}{3},
$$

so that the $\mathrm{H} \& \mathrm{~B}$ function (28) yields

$$
\frac{Y_{f}}{Y_{c}}=\left(s+\frac{m p}{Y_{c}}-\frac{m Y_{f}}{3 Y_{c}}\right)^{n}
$$

When $n=0.5$, Eq. (2.31) becomes a quadratic equation and the failure strength, $Y_{f}$, can be expressed in terms of pressure and unconfined compressive strength, $Y_{c}$, as

$$
Y_{f}=Y_{c}\left[\sqrt{s+\frac{m^{2}}{36}+\frac{m p}{Y_{c}}}-\frac{m}{6}\right]
$$

Eq. (2.32) may not be flexible enough to describe uniaxial strength both in compression and tension. Therefore the following, more general function is used

$$
Y_{f}(p)=\left\{\begin{array}{cc}
Y_{f 1}(p) & p>Y_{c} / 3 \\
Y_{f 2}(p)(1-\xi)+\xi Y_{f 1} & p \leq Y_{c} / 3
\end{array},\right.
$$

where $\xi$ is a linear function of pressure changing from zero at $p=-Y_{t} s^{n} / 3$ to one at $p=Y_{c} s^{n} / 3$.

The functions $Y_{f 1}$ and $Y_{f 2}$ are expressed as

$$
\begin{array}{r}
Y_{f 1}(p)=Y_{c}\left[\left(s+\frac{m^{2}}{36}+\frac{m p}{Y_{c}}\right)^{n}+s^{n}-\left(s+\frac{m^{2}}{36}+\frac{m}{3}\right)^{n}\right], \\
Y_{f 2}(p)=\frac{\left\langle p / Y_{c}+s \beta\right\rangle}{1 / 3+\beta}, \beta=\frac{2 R_{t}}{3\left(1-R_{t}\right)}, R_{t}=\frac{Y_{t}}{Y_{c} F_{L}(\beta)}
\end{array}
$$

Function $Y_{f 1}$ is used to describe the failure surface in compression and is derived as a genralized form of Hoek-Brown yield surface. The value of $Y_{c}$ for intact material can be found from unconfined compressive tests. Function $Y_{f 2}$ is used to match the unconfined tensile strength, where $F_{L}(\beta)$ is Lode function described later. Linear interpolation is used in Eq.2.33 to ensure continuous derivative with respect to pressure in the whole range as it is shown in Fig. 2.3. 


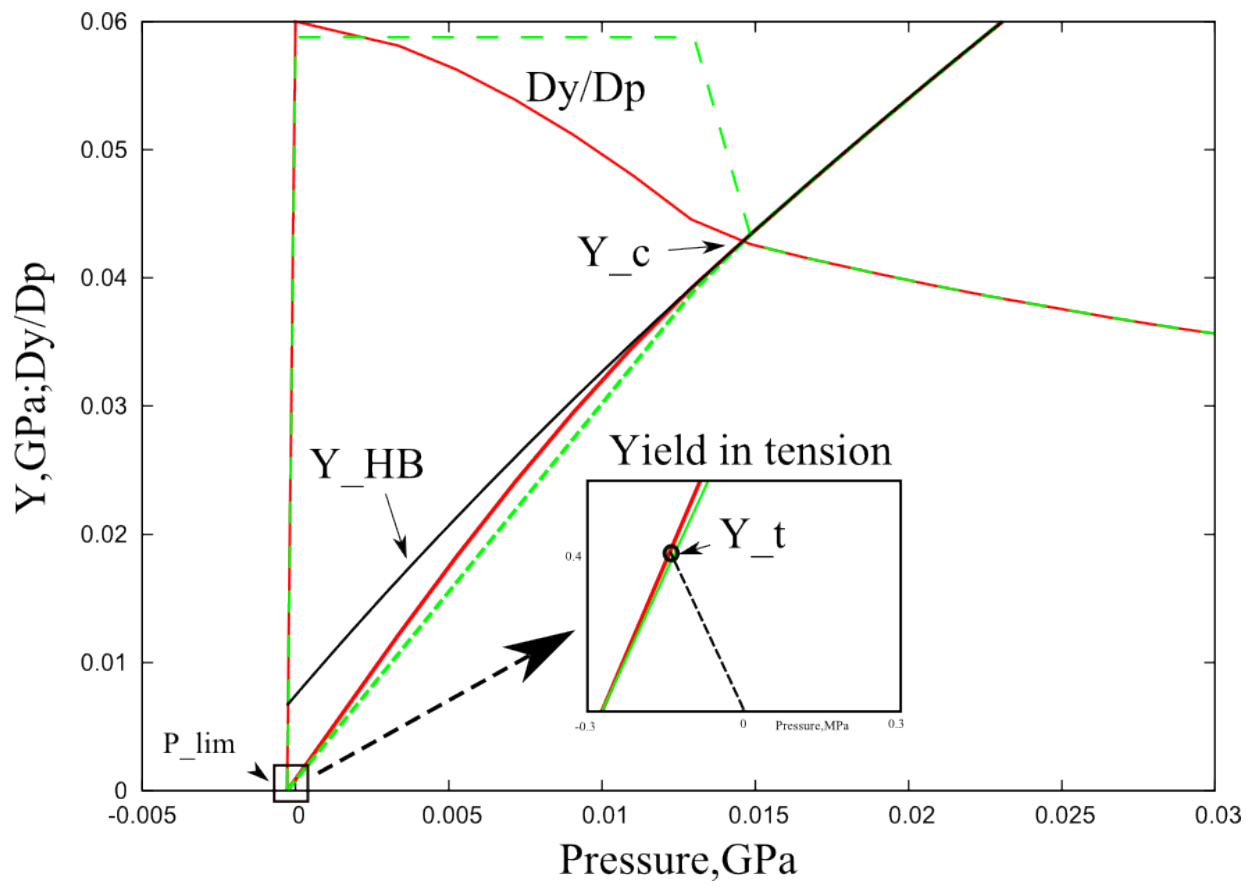

Figure 2.3: Yield surface and its derivative as a function of pressure. Dashed lines - model described in [12], solid lines - an improved model

Figure. 2.3 shows comparison between a pieswise-linear representation of the yield surface described in [12], and the current, improved model.

Both $Y_{c}$ and $Y_{t}$ can be parametrized in terms of the reference porosity $\Phi$ and the joint density.

According to "sliding wing crack theory"

$$
Y_{c}=\frac{\sqrt{6}}{\sqrt{1+\mu^{2}}-\mu} \frac{K_{I C}}{\sqrt{\pi c}},
$$

where $c$ is the average length of preexisting cracks [34]. It is reasonable to assume that $c$ is of the order of the average grain size. Generally, less porous rocks have smaller grain size, so the value of $Y_{c}$ decreases with porosity for the same type of rocks. The strength of sedimentary rocks has also been found to depend on other factors such as the amount of calcite, the texture, and pores distribution [35]. This means that for each data set one should use a specific correlation which takes these factors into consideration. A review of empirical correlations between strength and porosity can be found in [36].

The final yield surface accounting for pressure hardening, loading direction, softening due to microcracking (bulking), plastic strain hardening and thermal 
softening takes the form

$$
Y\left(\Phi, p, \beta, \Omega, \delta_{h}, \Theta\right)=Y_{T X C}(p) F_{\text {Lode }}(\beta) F_{\text {therm }}\left(J_{s}, \Theta\right),
$$

where $F_{\text {Lode }}(\beta)$ is a function of the Lode angle described in [23]. The thermal softening term, $F_{\text {therm }}\left(J_{s}, \Theta\right)$, can be expressed as

$$
F_{\text {therm }}\left(J_{s}, \Theta\right)=\frac{G\left(J_{s}, \Theta\right)}{G\left(J_{s}, \Theta=0\right)},
$$

where $\mathrm{G}$ is the shear modulus function of solid density and temperature The following simple function can be used

$$
G\left(J_{s}, \Theta\right)=G_{0}\left\langle 1-\Theta / \Theta_{\text {melt }}\left(J_{s}\right)\right\rangle,
$$

where $\Theta_{\text {melt }}\left(J_{s}\right)$ is supplied by the EOS.

Thermal softening effects can be important, for example, for problems involving shock waves. An EOS accounting for melting can be used to find the temperature, $\Theta$, using specific volume and thermal energy.

\subsection{Contact formulation and joint model}

Figure 2.4 shows two different subdomains $\Omega_{1}$ and $\Omega_{2}$ with external boundaries $\Gamma_{1}$ and $\Gamma_{2}$. Contacts may occur both between the boundary segments of the different domains (for example, between the points $B_{1}$ and $B_{2}$ or at point $C_{1}$ as shown in Fig. 2.4) as well as between the segments of the same boundary (for example, between the points $A_{1}$ and $A_{2}$ of boundary $\Gamma_{1}$ ). In contrast to a tra-

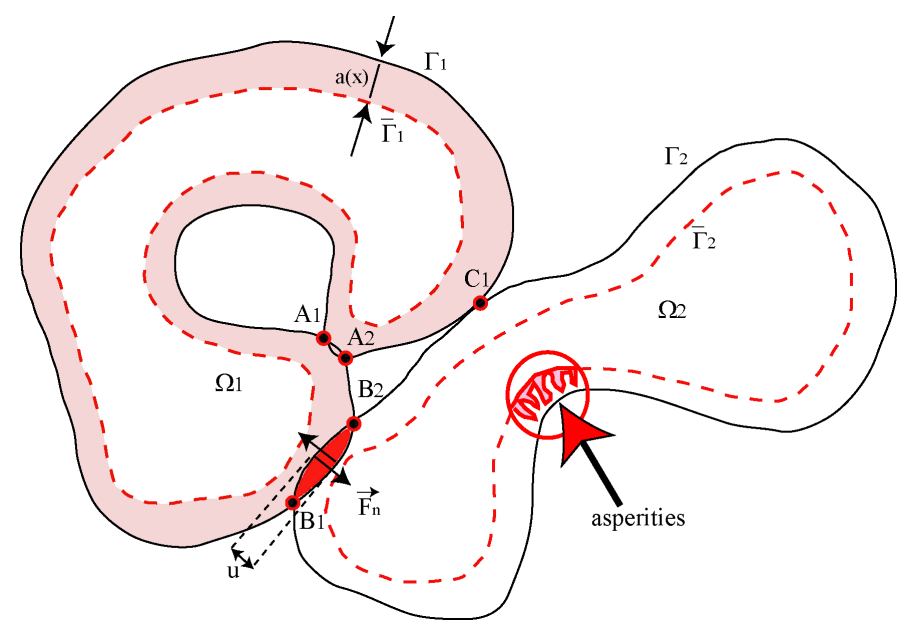

Figure 2.4: Contact of two domains with rough surfaces

ditional contact formulation, expressed as a impenetrability constrains between 


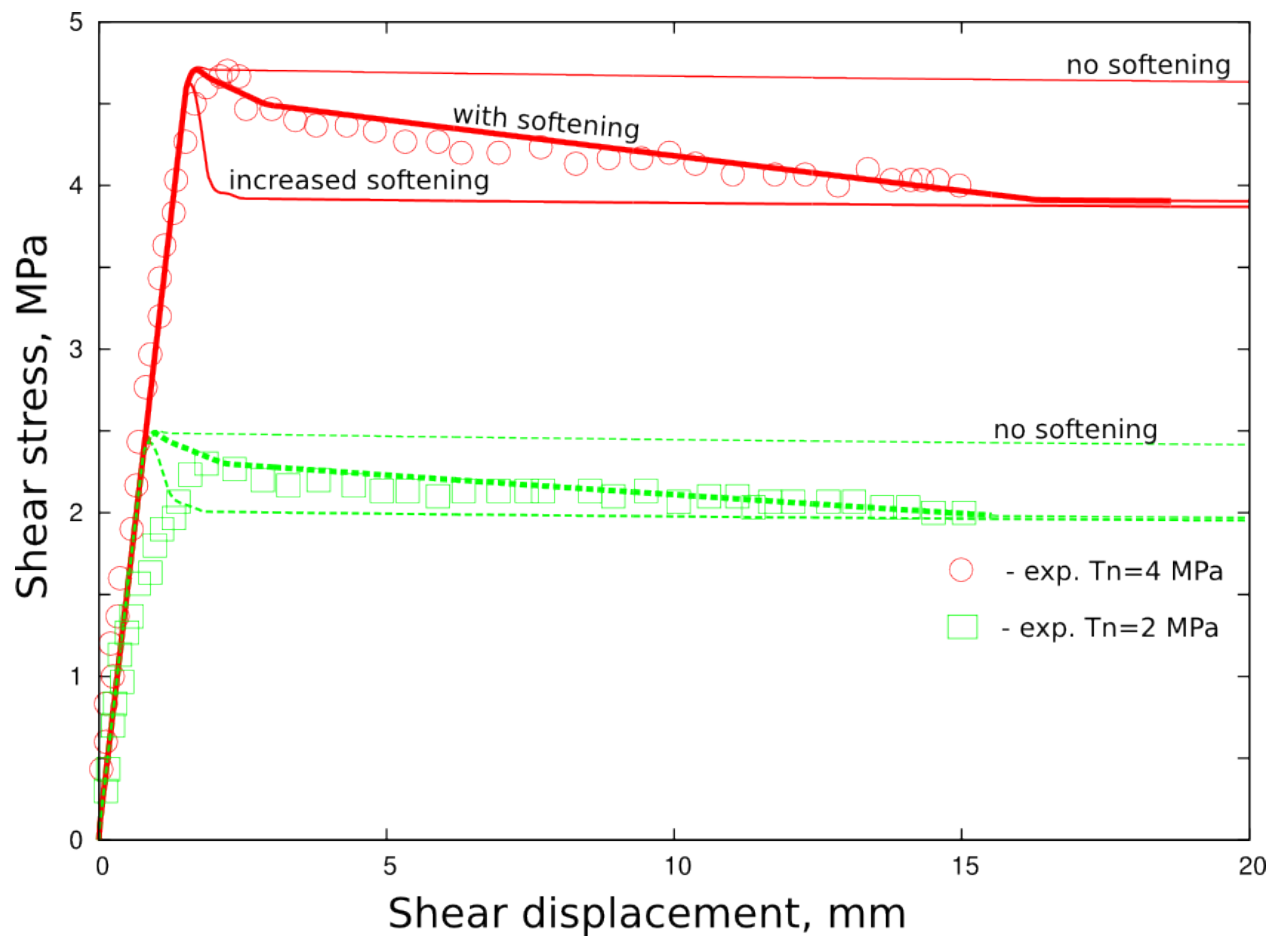

Figure 2.5: Shear stress versus shear displacement measured in experiments reported in [39] (shown with points) and calculated using current model (shown with lines) for two constant normal stresses $2 \mathrm{MPa}$ and $4 \mathrm{MPa}$. The critical shear slip, $v_{s p 0}$, was 15 for the base-line model and 0.5 for the increased softening case.

the contacted surfaces, the boundaries $\Gamma_{1}$ and $\Gamma_{2}$ are allowed to interpenetrate. Normal contact force at the boundary $F_{n}$ is a nonlinear function of of the interpenetration $u$. $F_{n}$ tends to infinity as $u \rightarrow a$. The aperture parameter, $a(\vec{x})$, characterizes the surface roughness and can vary along the surface. For smooth surfaces, $a \rightarrow 0$, and the current formulation reduces to a nonpenetration condition.

In the present formulation, contact surfaces are treated as finite boundary layers with special mechanical properties, different from ones used for the material in subdomains. These properties can be found either by undertaking a micro-mechanical study, where the surface asperities are modeled explicitly (see, for example, $[37,38])$, or by relying on the available empirical data.

Since the method described in this paper has been developed to model jointed rock media, the constitutive law for the contact is based on known empirical relationships for rock joints. Using the coordinate system associated with the joint, the stress at the boundary, $\sigma_{i j}$ can be decomposed into normal stress $\sigma_{n}$ 


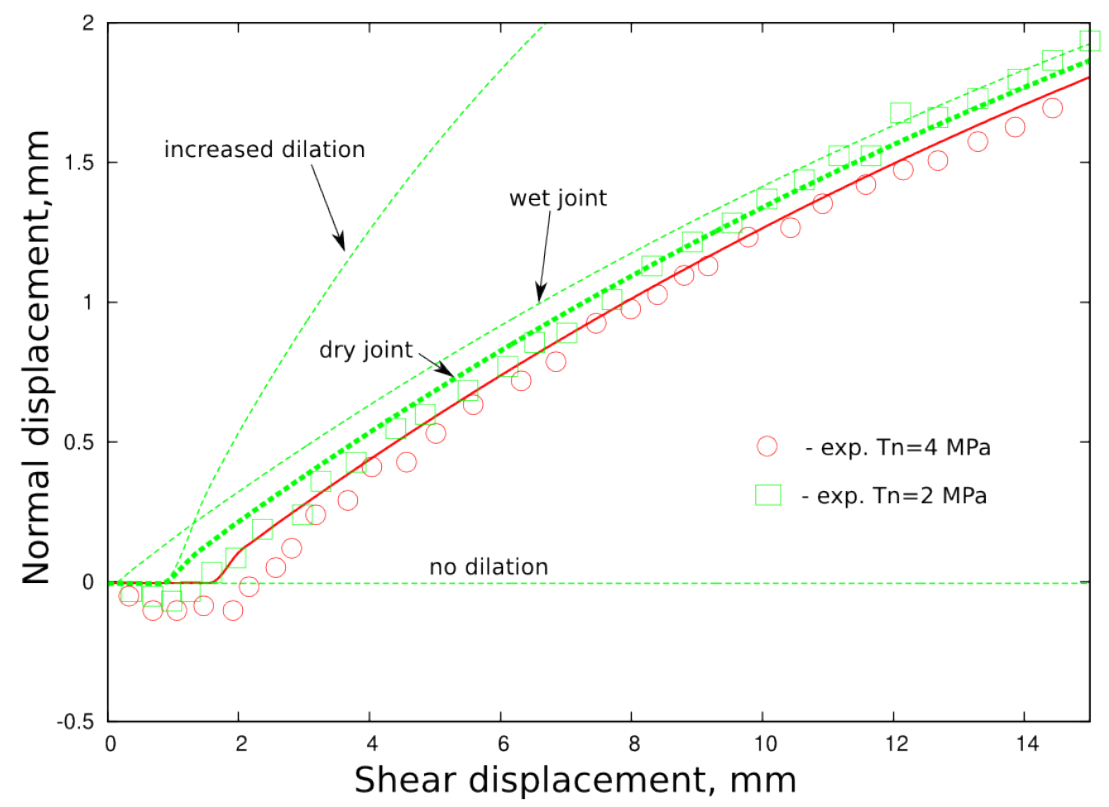

Figure 2.6: Normal displacement at the joint reported in [39] (shown with points) and calculated using current model (shown with lines) for two normal stresses $2 \mathrm{MPa}$ and $4 \mathrm{MPa}$. Dilation angle was 10 degrees for the base-line model and 25 degrees for case of the increased dilation. 
and shear stress vector $\tau_{i},(i=1,3)$ as

$$
\begin{array}{r}
\sigma_{n}=\sigma_{i j} n_{i} n_{j} \\
\tau_{i}=\sigma_{i j} n_{j}-\sigma_{n} n_{i},
\end{array}
$$

where $n_{i}$ is the outward normal vector.

The relative displacements of the joint surface, $u_{i}$, is decomposed into the shear displacement, $v_{i},(i=1,3)$ and the normal displacement, $u$.

$$
u_{i}=v_{i}+u n_{i}
$$

The displacements are divided into elastic and plastic parts as

$$
\begin{array}{r}
v_{i}=v_{i}^{e}+v_{i}^{p} \\
u=u^{e}+u^{p}
\end{array}
$$

The stresses evolve in time as:

$$
\begin{array}{r}
\dot{\sigma_{n}}=\frac{E(u)}{a} \dot{u^{e}} \\
\dot{\tau_{i}}=\frac{G}{a} \dot{v_{i}},
\end{array}
$$

where $\mathrm{G}$ is the shear modulus for the joint, $\mathrm{E}$ is the normal modulus of the joint,which is a function of the normal displacement.

It is known from experimental observations [40] that joint closure, $u$, is a nonlinear function of the applied normal stress, resembling a hyperbola. Therefore, the following functional form is used for the normal modulus, $E(u)$

$$
E= \begin{cases}E_{0} \frac{a^{2}}{(a-u)^{2}} & \dot{u}>0 \\ E_{0} \frac{a^{2}}{\left(a-u_{\max }\right)^{2}} & \dot{u} \leq 0\end{cases}
$$

In Eq.(2.47) $a$ is the aperture, $u$ is the normal closure, $E_{0}$ is the initial normal modulus and $u_{\max }$ is the maximum closure up to the current time. During the unloading, $\dot{u}<0$, the stiffness defined by the value of $u_{\max }$ remains constant.

Plastic parts of the displacements, $v_{i}{ }^{p}$ and $u^{p}$, can be found using a flow rule. It is known that shear behavior of rock joints exhibits nonassociative response [41]. Therefore, it is common to use a nonassociative flow rule adopting a plastic potential, $Q$, similar to the Morh-Coulomb slip function, $F$, used to limit the shear stress but with a different friction coefficient, $\mu_{\Psi}=\tan (\Psi)$ related to the angle of dilation, $\Psi$. The functions $F$ and $Q$ are written as

$$
\begin{array}{r}
F=|\tau|-\tau_{\max } \\
Q=|\tau|-\sigma_{n}{ }^{e f f} \mu_{\Psi},
\end{array}
$$


where $\sigma_{n}{ }^{e f f}$ is the effective stress expressed as $\sigma_{n}{ }^{e f f}=\left\langle\sigma_{n}-P_{f}\right\rangle$ and $\tau_{\max }$ is the maximum shear stress written as

$$
\tau_{\max }=C\left(v_{s p}\right)+{\sigma_{n}}^{e f f} \mu
$$

In Eq.(2.50) $\mathrm{C}$ is the shear cohesion which depends on plastic slip $v_{s p}$ described later, and $\mu=\tan (\phi)$ is the friction coefficient related to the friction angle $\phi$. The fluid pressure, $P_{f}$, is to describe the effect of the fluid for wet joints. Using the flow rule the increment of the plastic shear displacement, $d v_{i}{ }^{p}$ can be found as

$$
d v_{i}^{p}= \begin{cases}0, & F<0 \\ \lambda \frac{\partial Q}{\partial \tau_{i}}, & F \geq 0\end{cases}
$$

where $\lambda$ is a scalar multiplier determined by requiring that the new stress is located on the slip surface $(F=0)$. Using $(2.51)$ the increment of the magnitude of the plastic shear displacement, called here the shear slip and defined as $v_{s p}=$ $\sqrt{v_{i} v_{i}}$, is expressed as

$$
\begin{array}{r}
d v_{s p}=\lambda \\
\lambda=\frac{\left\langle|\tau|-\tau_{\max }\right\rangle}{G},
\end{array}
$$

where \langle\rangle are McAuley brackets.

The friction coefficient $\mu$ changes with the amount of shear slip from the initial value of $\mu_{0}$ to the residual value of $\mu_{1}$ due to the softening effect as

$$
\mu=\mu_{1}+\left(\mu_{0}-\mu_{1}\right)\left\langle 1-v_{s p} / v_{s p 0}\right\rangle,
$$

where $v_{s p 0}$ is the value of the shear slip corresponding to complete softening.

Both the tensile strength and the friction slope may change due to damage caused by shear slip. The friction slope changes with shear slip according to Eq.(2.54). Thus, it is convenient to define the cohesion, $C$, used in Eq.(2.50) as a product of the tensile strength of the joint, $\sigma_{t}$ to the friction slope $\mu$. The tensile strength decreases with shear slip until it reaches zero as

$$
\sigma_{t}=\left\langle\sigma_{t 0}-\alpha v_{s p}\right\rangle,
$$

where $\sigma_{t 0}$ is the initial value of the tensile strength and $\alpha$ is the rate of softening.

If $\lambda>0$, then the increment of normal plastic displacement is found as

$$
d u^{p}=-\lambda \frac{\partial Q}{\partial \sigma_{n}}=\lambda\left(\mu_{\Psi}+\sigma_{n}{ }^{e f f} \frac{\partial \mu_{\Psi}}{\partial \sigma_{n}}\right)
$$

It is known from shear test experiments that joint dilation decreases with confinement. To account for this effect a simple linear dependence on the effective normal stress is used [42].

$$
\mu_{\Psi}=\mu_{\Psi 0}\left\langle 1-\sigma_{n}^{e f f} / \sigma_{c r}\right\rangle
$$


where $\mu_{\Psi 0}$ is the dilation coefficient at zero normal stress and $\sigma_{c r}$ is the critical effective stress above which dilation will not occur. Also, to limit dilation to $u_{\max }^{p}$ the dilation coefficient $\mu_{\Psi 0}$ depends on the normal plastic displacement as

$$
\mu_{\Psi 0}\left(u^{p}\right)=\mu_{\Psi 00}\left\langle 1+u^{p} / u_{\max }^{p}\right\rangle,
$$

where $\mu_{\Psi 00}$ is the initial dilation coefficient and $0<u^{p}{ }_{\max }<a$. Note, that $u^{p}$ is negative because it describes the opening of the joint due to dilation while $u^{p} \max _{\text {ix }}$ is positive. Using Eq.(2.56), Eq.(2.57) and Eq.(2.58) gives the following equation to find the new plastic normal displacement

$$
\dot{u}^{p}=-\mu_{\Psi 00}\left\langle 1+u^{p} / u_{\text {max }}^{p}\right\rangle \beta \dot{u}_{s p}, \beta=\left\langle 1-\frac{2 \sigma_{n}^{e f f}}{\sigma_{c r}}\right\rangle
$$

Solving Eq.( 2.59) gives the following expression for the plastic normal displacement increment

$$
d u^{p}=\left\langle u_{\max }^{p}+u^{p}\right\rangle\left(\exp \left(-\frac{\beta \mu_{\Psi_{00}} d v_{s p}}{u_{\max }^{p}}-1\right)\right) \approx-\beta \mu_{\Psi_{0}} d v_{s p}
$$

In the dynamic case, the mass of the fluid in the joint is assumed to be unchanged, so that the fluid will react to the volume change associated with the joint opening due to dilation as

$$
d P_{f}=K_{f l} \frac{d u^{p}}{a} \beta \mu_{\Psi_{0}} d v_{s p},
$$

where $K_{f l}$ effective bulk modulus of the fluid.

If the normal displacement is constrained $(d u=0)$, the normal stress increment due to the shear slip can be written as

$$
d \sigma_{n}=E\left(d u-d u^{p}\right)=-E d u^{p} \approx E \beta \mu_{\Psi_{0}} d v_{s p}
$$

To satisfy the second law of thermodynamics the dissipative work $d W^{p}$ defined as $\sigma_{i} d u_{i}{ }^{p}$ should be positive. The plastic work can be written as

$$
d W^{p}=\sigma_{n} d u^{p}+\tau d v_{s p}=-\sigma_{n} \beta \mu_{\Psi_{0}} d v_{s p}+\tau d v_{s p} \geq 0
$$

The first term in Eq. ( 2.63) is negative and the second one is positive. Since the shear stress $\tau>\mu \sigma_{n}, \beta<1$ and $\mu_{\Psi}<\mu$ the net plastic work is always positive which is consistent with the second law of thermodynamics.

To validate the model shear test experiments for granite joints [39] were simulated using a driver routine, which integrates the equations of motion described above. Results of the simulations are shown in Fig. 2.5 and Fig. 2.6 It is seen from Fig. 2.5 that frictional softening is needed to described the experiments. Also, right amount of dilation (controlled by the dilation coefficient) is required to describe the normal displacement evolution observed in the tests. Due to dilation coefficient dependency on the normal stress described by Eq. 2.57, less dilation is calculated at high normal stress, which is also observed in the experiments. It is seen from Fig. 2.6, that dilation slows with the increase of $u^{p}$. 
This effect is described in the model by reducing the dilation coefficient with the amount of the dilation in Eq. 2.58.

Wet joints where the fluid pressure is controlled $\left(P_{f}=0.5 \mathrm{MPa}\right)$ show an increased dilation effect, which was also observed in the experiments [39].

\subsection{Meshing Representative Volumes for jointed rock}

Discrete 3D models consisting of blocks of rock and joints with the given properties can be built using parameters, which can be found from rock mass observation and characterization, such as joint spacing, orientation and persistence. Each rock block can be subdiscretized into either hexahedral or tetrahedral finite elements. The joints are modeled using advanced contact algorithm with history variables described above, where the mesh boundaries are aligned with the joints.

\subsubsection{Two and Three joint set model}

Very often, the joints are present in the rock mass as joint sets. To model this situation a meshing algorithm was developed which paves the space with parallelepiped blocks. Figure 2.7 illustrates how two joint set with arbitrary persistency can be represented in $2 \mathrm{D}$ case. First, the space is paved with the blocks of the same size. Then the sliding contacts are set where the joints are persistent (shown with dots) and cohesive elements with high value for the cohesion (comparable to the material cohesion) is set everywhere else.

\subsubsection{Random joint model}

When persistent joints are randomly distributed in the rock they will cut the continuum rock mass into an assembly of polyhedral blocks Meshing such system can be a challenging task since some blocks can be either very small or have short edges which will result into a small elements. Using such small elements in FE analysis will result in a very stiff limitations on time step dictated by stability constraints (such as Courant condition). An algorithm to mesh a system of polyhedral blocks with controlled distribution of block sizes was developed. The algorithm is based on a packing of premeshed spheres within given volume with consequent stretching of spheres to close the gaps between them. Figure.2.8 illustrates the main steps of meshing algorithm in 2D.

The external nodes are projected onto the boundaries of Voronoi cells created by the packed spheres. Because of a finite discretization on the boundaries there is no guarantee that any node will be projected into the corners of Voronoi cells. An algorithm in 2D was developed which moves the nearest node to the nearest corner to close the gaps between the spheres. Equipotantial mesh smoothing can be applied to improve mesh quality after mesh stretching and node dis- 


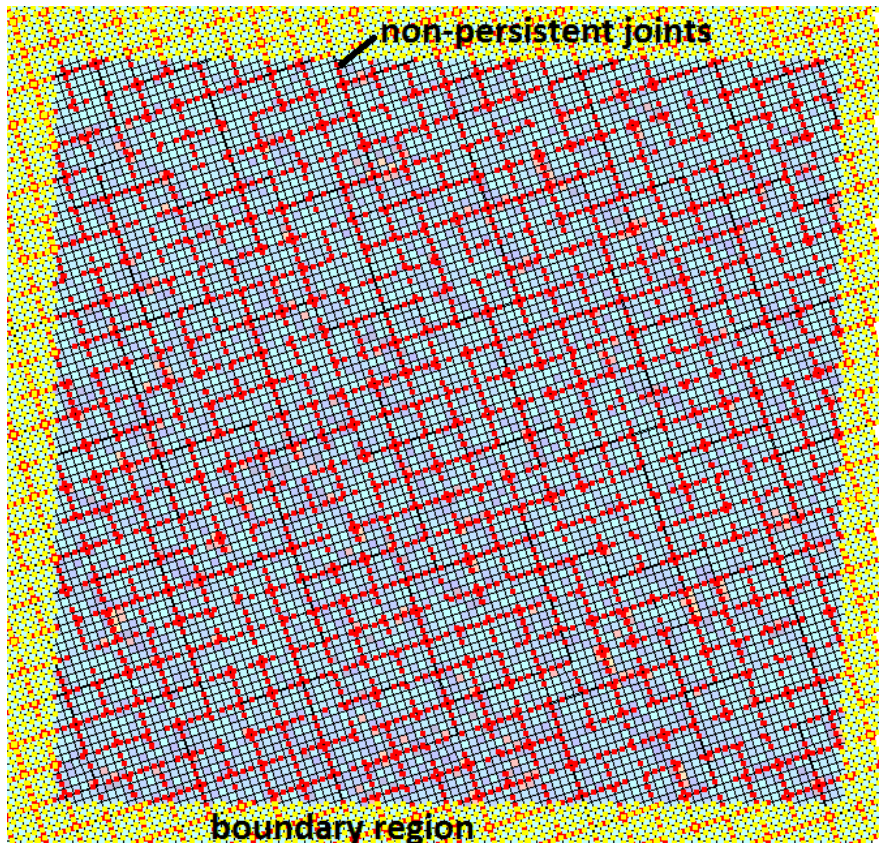

Figure 2.7: Meshing representative volume with two joint sets

placements at the boundaries. Figure.2.9 illustrates the meshing algorithm in $3 \mathrm{D}$.

\subsection{Meso scale studies for jointed rock masses}

\subsubsection{Scaling the yield surface for jointed rocks}

The meshing algorithm described above can be applied to guide the development of better continuum models for jointed rock mass. Figure.2.10 shows stress evolution in a representative volume of randomly jointed granite. meshing technique based on packed spheres described above was used to mesh the volume. Velocity boundary conditions were applied along the external surface corresponding to an arbitrary uniform deformation of the volume. To achieve some initial confinement the volume was first hydrostatically prestressed, then a shear deformation combined with either an extension or compression was applied to increase the deviatoric stress. As the blocks deform under shear loading, voids open at the contacts between the blocks causing build up of the hydrostatic pressure (dilatancy effect). Deviatoric stress is limited both by the plastic deformations within the blocks as well as by sliding at the block boundaries. Both these processes define the ultimate strength of the jointed system which is always less than the strength of the intact rock. 


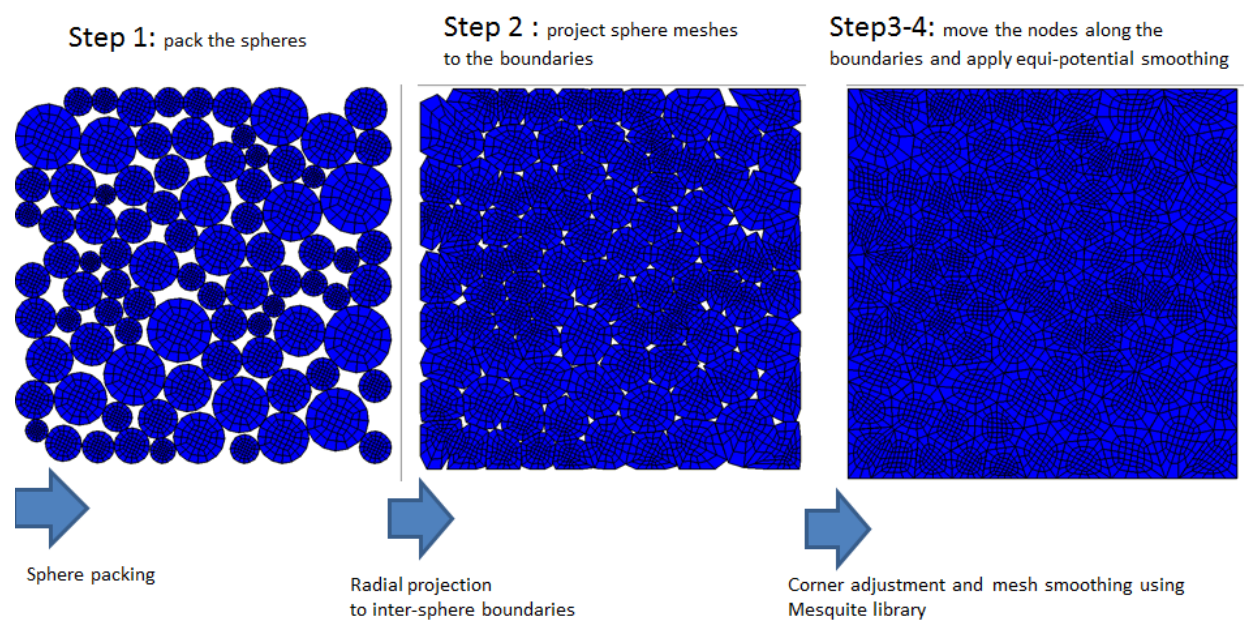

Figure 2.8: Main steps for multiblock meshing

It is known that jointed rock mass has properties significantly different from ones of the intact rock. Traditional scaling approach used in rock engineering uses Hoek-Brown scaling rule which was built intu the current model. Yet, these synthetic simulations of triaxial loading tests show that there is no good GSI index to match rock mass response both at low stress confinement (used in rock mechanics) and high confinements realized in shock waves. One may conclude that to scale the continuum model to the rock masses for shock wave applications one must use a different scaling rule.

\subsubsection{Poroelasticity of jointed rocks}

Presence of joints can also change the poroelastic properties of the rock mass compared to the intact rock. The simplest way to account for this effect is to assume that joints are linear as well as the material itself and do not interact with each other [43]. Effects of the joints on both P-wave and S-wave speeds were studied experimentally in [44].

The following formula can be derived for the effective bulk modulus if joints are random.

$$
K_{e f f}=\frac{E_{s}}{9}\left(\frac{3(1+\nu) s_{j} E_{j} / a+2 E_{s}}{(1+\nu)(1-2 \nu) s_{j} E_{j} / a+(1-\nu) E_{s}}\right)
$$

Figure 2.11 shows results of hydrostatic compression of a RVE volume with three sets of joints. Straight lines correspond to effective linear media found using formular 2.64. It is seen that the formula captures initial slope pretty well if the joints are persistent. The size of the RVE was 10x10x10 m with the joint spacing and orientation corresponding to one found at the SPE site described 


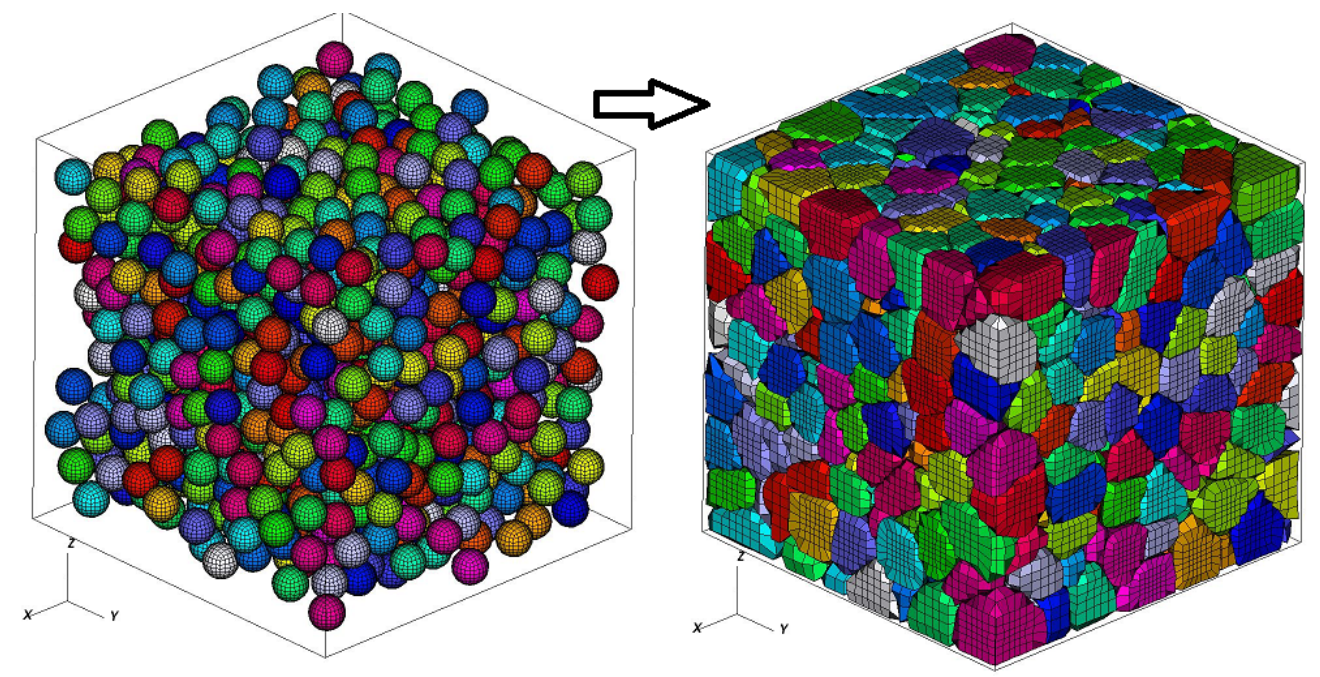

Figure 2.9: 3D multiblock meshing is derived from sphere packing

later. Both joint aperture, $a$, and normal stiffness, $E_{j}$, effect the effective bulk modulus, $K_{\text {eff }}$. The joint spacing, $s_{j}$, was assumed to be $1.25 \mathrm{~m}$.

Poroelasticity parameter, $a$, used in Eq.2.9 can then be evaluated as

$$
a\left(\Phi, s_{j}\right)=1-\left(1-a_{0}(\Phi)\right) \sqrt{\frac{K_{s}}{K_{e f f}}},
$$

where $a_{0}(\Phi)$ is poroelasticity parameter for small rock samples and $s_{j}$ is the joint spacing.

Even though using $a$ defined by Eq.2.65 provides right initial bulk modulus it may not provide right curvature. Figure 2.12 shows pressure volume curves versus material driver results (dashed lines) calculated using parameter $a$ from Eq.2.65. For low joint density (case C) agreement is satisfactory, but for high joint densities (case A) effective poroelasticity model where $a$ is calculated by Eq.2.65 underestimates poroelasticity effect. If joints are not persistent effect of the joints calculated using the formula 2.64 may change. Figure 2.13 shows how poroelasticity will decrease with decreased persistency. Since in reality joints are rarely fully persistent underestimation of the poroelasticity can be compensated by the effect of poroelasticity reduction due to low persistency. Thus, for the case A which is not described well for fully persistent joints satisfactory agreement is produced if the joints are not persistent, as it is shown in Fig. 2.13 for $70 \%$ persistent joints (case II). Effective aperture used in Eq.2.64 to find the effective bulk modulus which is then used in Eq.2.65 is scaled by persistency to reduce the volume occupied by the joints. 


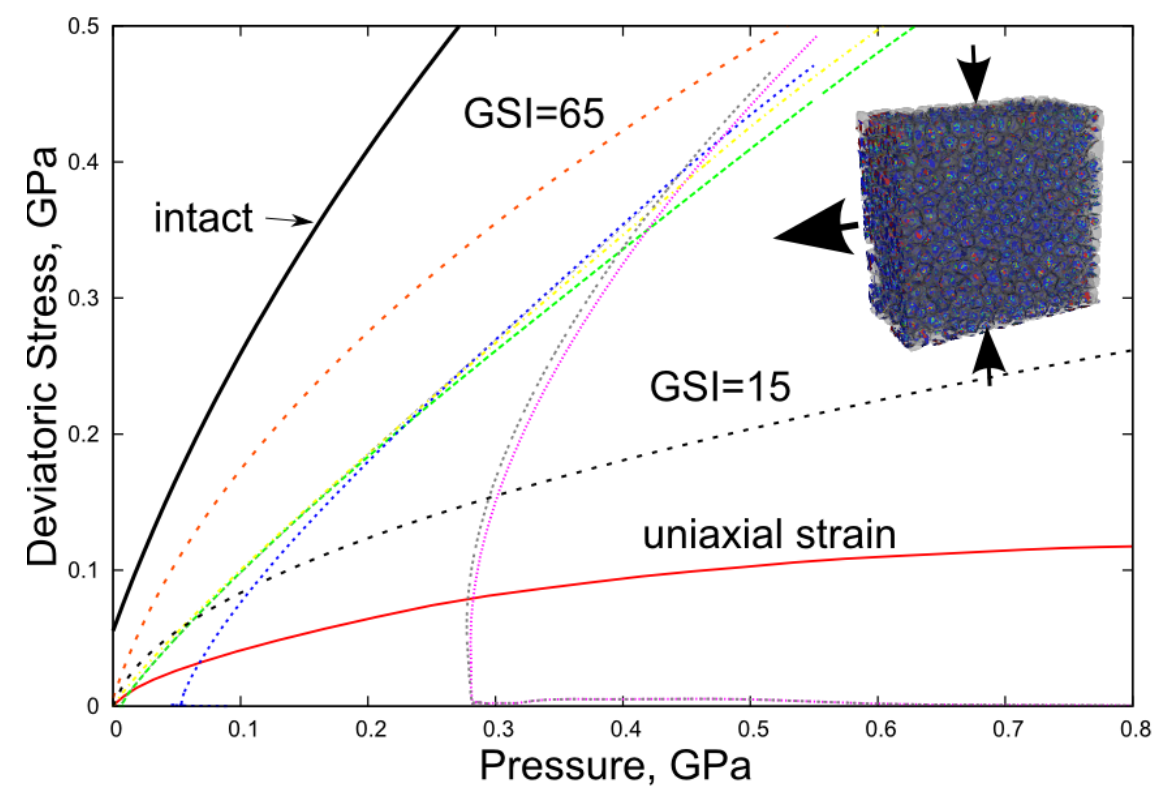

Figure 2.10: Stress evolution calculated for a representative volume with granite blocks packed using the method described above. Thick solid line shows the yield surface for the intact granite, dashed lines show scaled models for two values of GSI index using traditional approach and dotted lines show triaxial loading from various confinements, thin solid line shows results of uniaxial strain loading. 


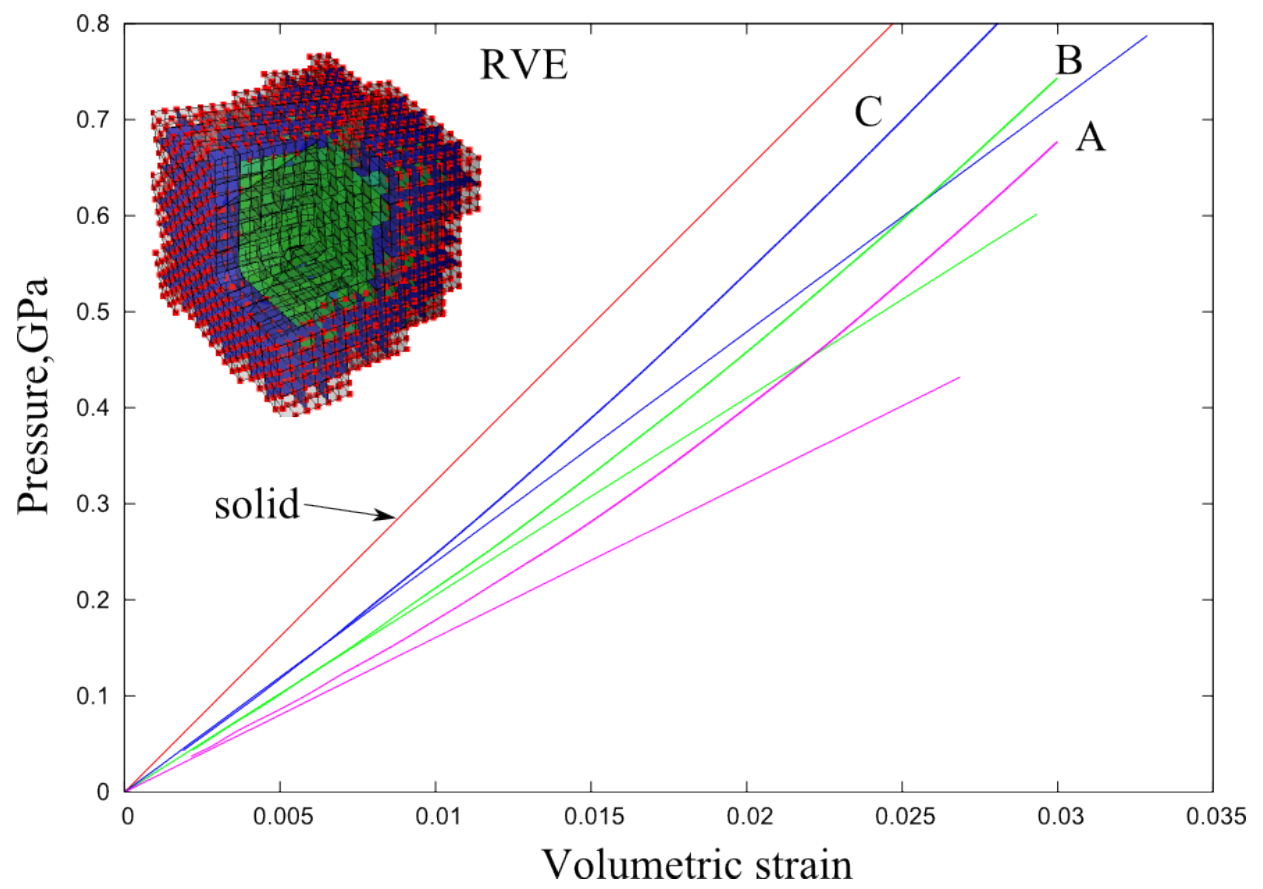

Figure 2.11: Pressure volume response in hydrostatic compression with different joint apertures. Fully persistent joints were assumed, A- $a=10 \mathrm{~mm}, E_{j}=0.1 \mathrm{GPa}$; $\mathrm{B}-a=10 \mathrm{~mm}, E_{j}=0.3 \mathrm{GPa}$; $\mathrm{C}-a=5 \mathrm{~mm}, E_{j}=0.3 \mathrm{GPa}$ 


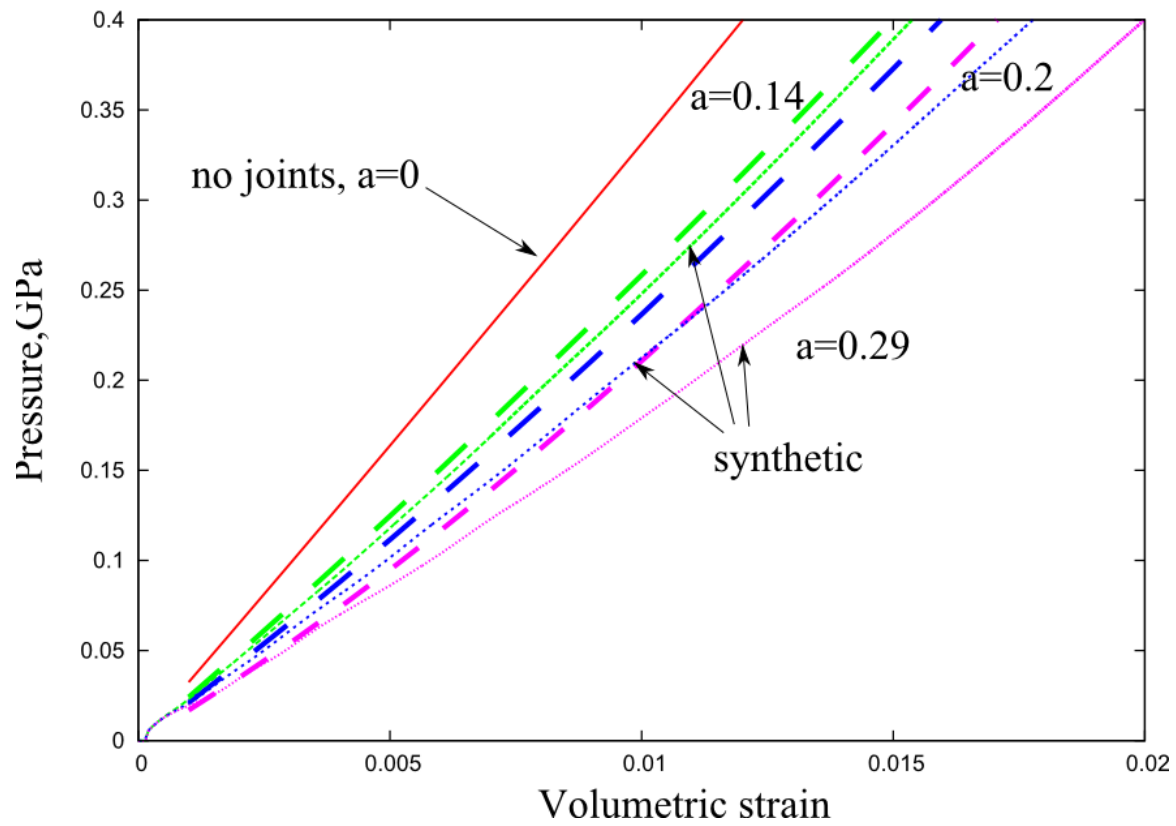

Figure 2.12: Comparison of synthetic P-V curves versus material responses calculated using effective $a$ (dashed curves) 


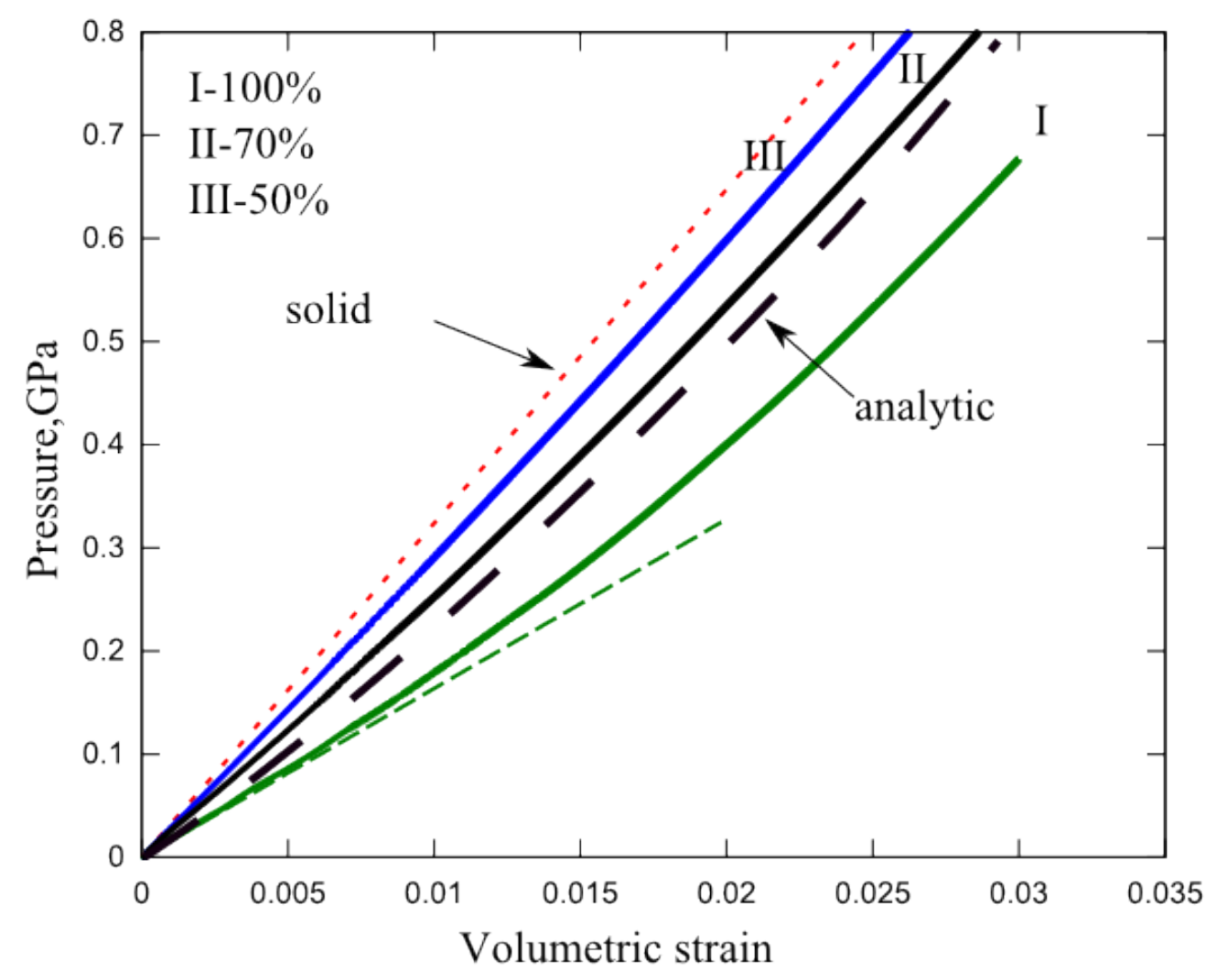

Figure 2.13: Pressure volume response in hydrostatic compression for different joint persistency. I-100\%,II-70\%, III-50\%. Dashed line correspond to the effective model where parameter $a$ is calculated using Eq.2.65 with aperture scaled by persistency 


\section{Chapter 3}

\section{Discrete-Continuum methods for shock waves in jointed rock masses}

\subsection{Hybrid element solvers}

A framework was developed to use multiple element solvers either sequentially or concurrently. For example, to model kinematics of structural collapse due to gravity one must resolve a characteristic time of $\sqrt{2 H / g}$ which is typically much more than the time step required for stable integration in explicit compressible FE solver $H / c$, where $c$ is the material sound speed and $H$ is a characteristic size of the structure. Therefore, converting element solver to a rigid body solver, which does not have strict time step limitation, at late time would save computational time. Figure 3.1 illustrate how this hybrid approach can be applied to a tunnel collapse problem. The rock formation was modelled as an assembly of rock blocks. Each block was subdiscretized into finite elements. The blocks around the source, where compressibility of the rock is important, were treated as compressible using $\mathrm{FE}$ as it is shown in the figure. At later times, when the wave passes the tunnel, block solver is switched to DEM to model kinematics of the block motion as it is shown in Fig. 3.2

\subsection{Hybrid contact solver}

\subsubsection{Advection of history variables at the contacts}

Hystory variables at the contact are stored both at the faces involved in contact and the common planes (contact elements described in [11]). Anytime a history variable is increamented at the common plane, the same history variable is also increamented at the faces. Thus, if a face slides past another face the 


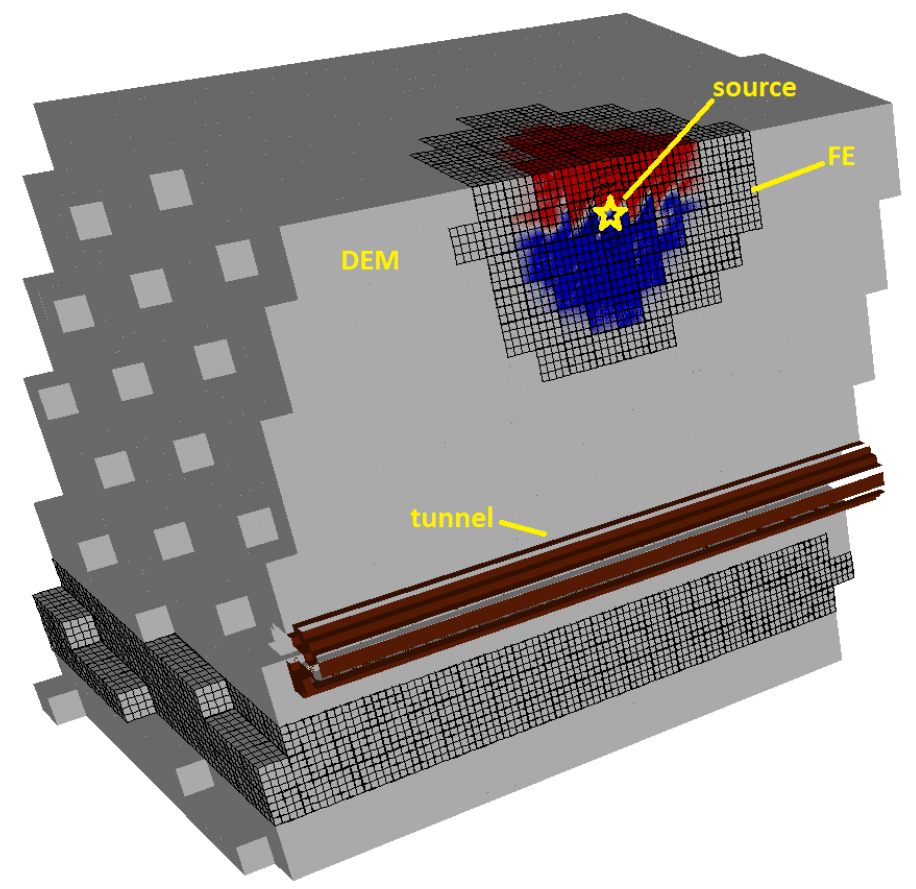

Figure 3.1: Tunnel collapse caused by a an explosion. Mesh is shown for the blocks where Finite element solver is applied, the rest is computed by discret element solver. Contours of vertical velocity is also shown.

contact between these two faces disappears, but the face accounts for the history evolution done at this common plane. When this face meets a new face and creates a new contact (common plane) the initial values for the history variables are taken as an average between the values at the faces. The stress state (normal and shear stress) is initialize as a projection from the average element stress at the adjacent elements. Therefore creation of new contacts at a sliding interface which is under some normal stress does not cause any stress drop in normal stress.

\subsubsection{Efficient search algorithm for hybrid contacts}

Presence of multiple contacts can make calculations computationally expensive. The most expensive part of the calculations with multiple contacts could be due to frequent search of the new contacts. But very often during dynamic loading of jointed systems only a small number of contact surfaces remains active at any time. Therefore, most of contact faces do not change connectivity over many computational cycles. Because of that, they can be excluded from new contact search. Thus, contact faces should only be added to the list of candidates which 

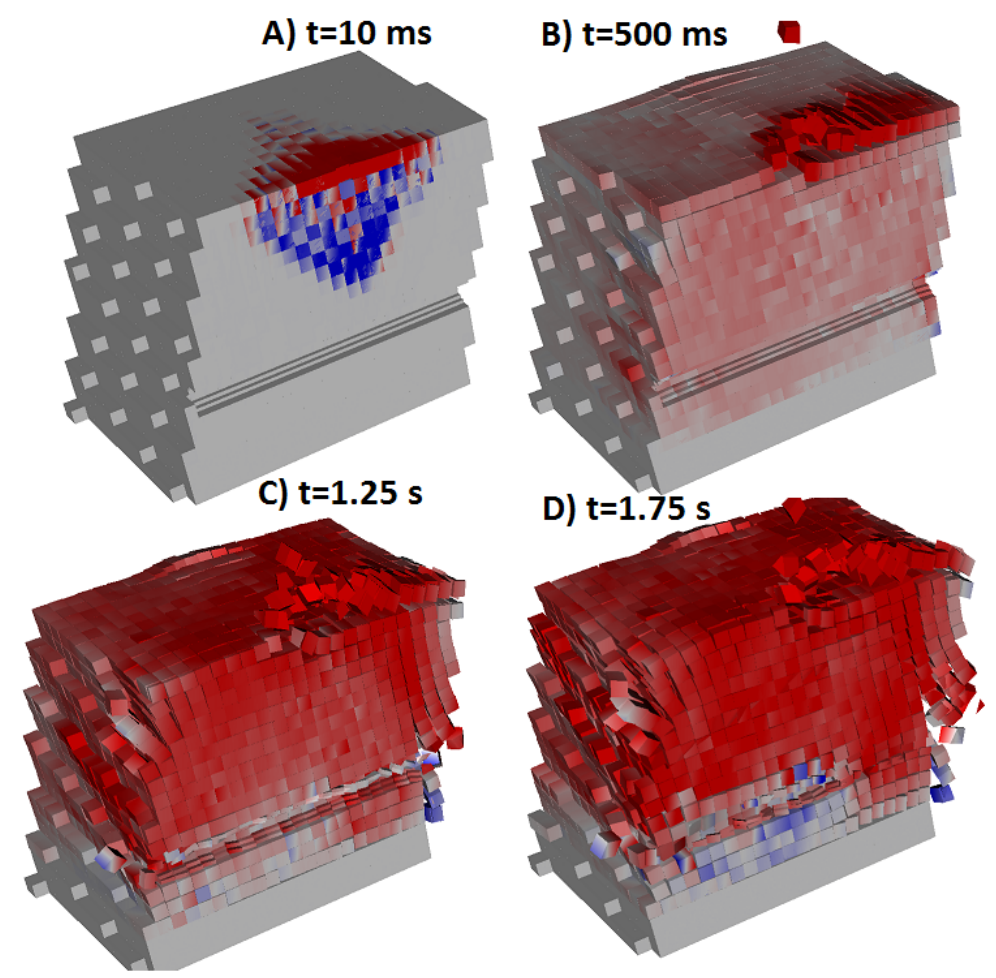

Figure 3.2: Vertical velocity contours and block motion at late times computed by DE solver

can create new contacts if there is a significant shear displacement taking place at these faces. We have introduced two types of contacts: cohesive contacts and collisional contacts which are initially defined by the boundary conditions set at the faces. Collisional contacts (called here type 0 contacts) are assumed to change dynamically every few cycles and are typically assigned to the external boundaries of discrete blocks (such as, for example sand grains or moving objects). Cohesive contacts in turn (type 1) are assumed to stay in place until a certain amount of shear slip is accumulated at the faces. When it happens, the contact can be considered broken and its type is switched to type 0 (collisional contacts). All faces of type 1 contact are excluded from the contact search once the contacts involving those faces are established. Type 0 contact faces are generally included into new contact search.

To improve the computational efficiency further, type 0 contact faces can be temporarily excluded from the new contact search if they remain in full contact (more than $90 \%$ of faces area is involved into contacts with other faces) and average shear displacement relative to the faces they contact are small, which 
means that they do not have a chance to move and create new contacts. Once this displacement reaches a critical value (set by the user) faces are tagged to be sorted into bins for new contact search and the history variable (relative average shear displacement) is reset to zero. After new contact search these faces are hidden from the future searches until they accumulate critical shear displacement. Figure 3.3 shows an example of using hybrid contact algorithm

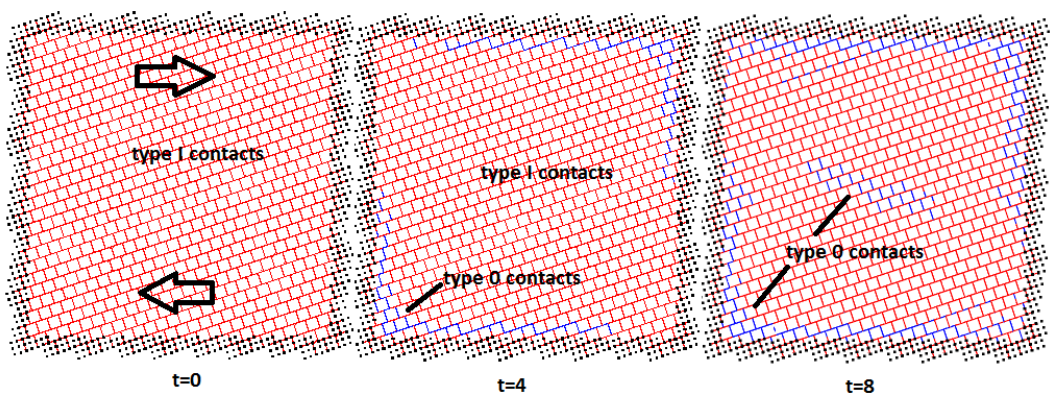

Figure 3.3: Contact type evolution for 2D shear loading of a jointed rock volume

for shear loading of a representative jointed region in 2D. A jointed system with two sets of joints was loaded using velocity boundary conditions (shown with black dots) applied to the nodes of the elements on the periphery of the region. Plastic slip has developed overtime at the contacts close to the boundaries and in the middle of the region which triggered the transition from type 1 contact to type 0 contact. The problem runs roughly two times faster when hybrid contacts are used.

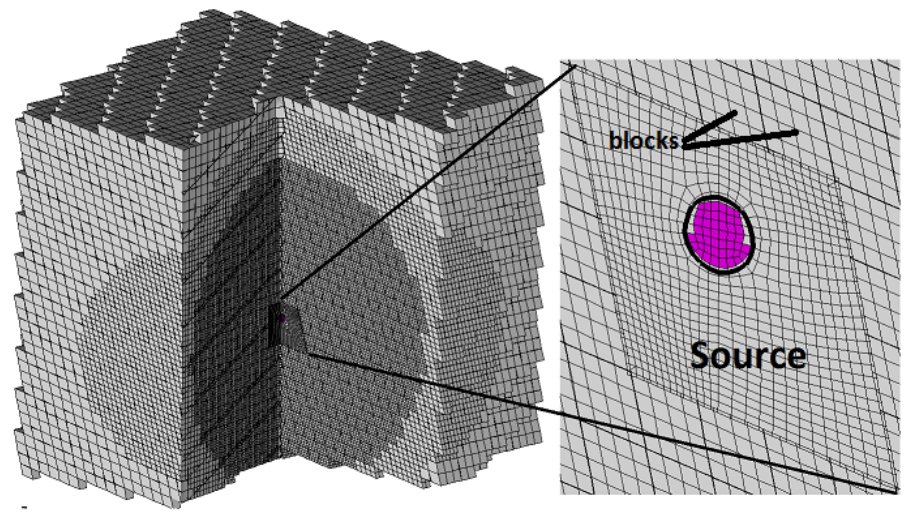

Figure 3.4: Mesh and block locations for 3D explosion in a jointed rock

Figure 3.4 shows problem set-up for calculation of spherical explosion in a 3 set jointed rock formation. The region was meshes as an assembly of paralelip- 
iped blocks subdiscretize into different level of accuracy. The source block was meshed differently using conforming hexahedral meshes for a sphere and a block with a spherical cavity of the same size. The radius of the was $0.625 \mathrm{~m}$, and the size of the source block was 4 times bigger than the other blocks. The source was modeled as an ideal gas material with density of $1.32 \mathrm{~g} / \mathrm{cc}$, specific internal energy of $3.9 \mathrm{~kJ} / \mathrm{g}$ and gamma parameter of 1.3. Cohesive contacts (type 1) were used between the block which later transitioned into collisional contacts (type 0) as it is shown in Figure 3.5, which also shows the pressure contours in the range (0.001-0.01 GPa).

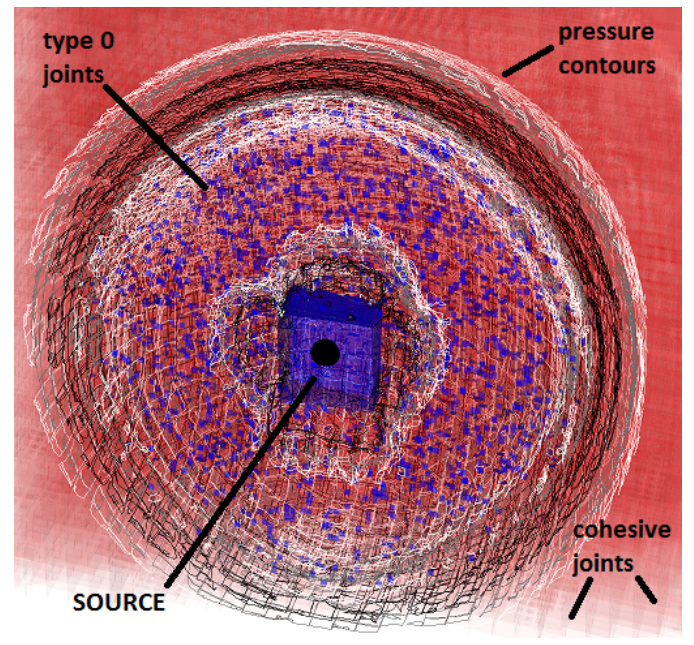

Figure 3.5: Pressure contours and joint types at time $\mathrm{t}=3 \mathrm{~ms}$

\subsection{Discrete modeling of fracture}

To model damage and fragmentation in rock masses one can either use a continuum plasticity model which includes some history variables describing the strength softening or allow an explicit description of new fractures in the material at localized zones of failure which can be significantly smaller than the cell size. The first, continuum approach, can be mesh sensitive, since sub-cell localization zones cannot be accurately resolved. The second approach includes algorithmic difficulties to transition from continuum to discrete description of the failure zone. We believe that when new localized failure zones are created (such as cracks, joints) mechanical properties in those zones (such as friction, dilatancy, stiffness) are significantly different from the intact material, and, therefore, it is difficult to described them using the same strength model as the one used for the intact material. Even if such a wide-range strength continuum model is designed one should resolve the localization zone which could be 


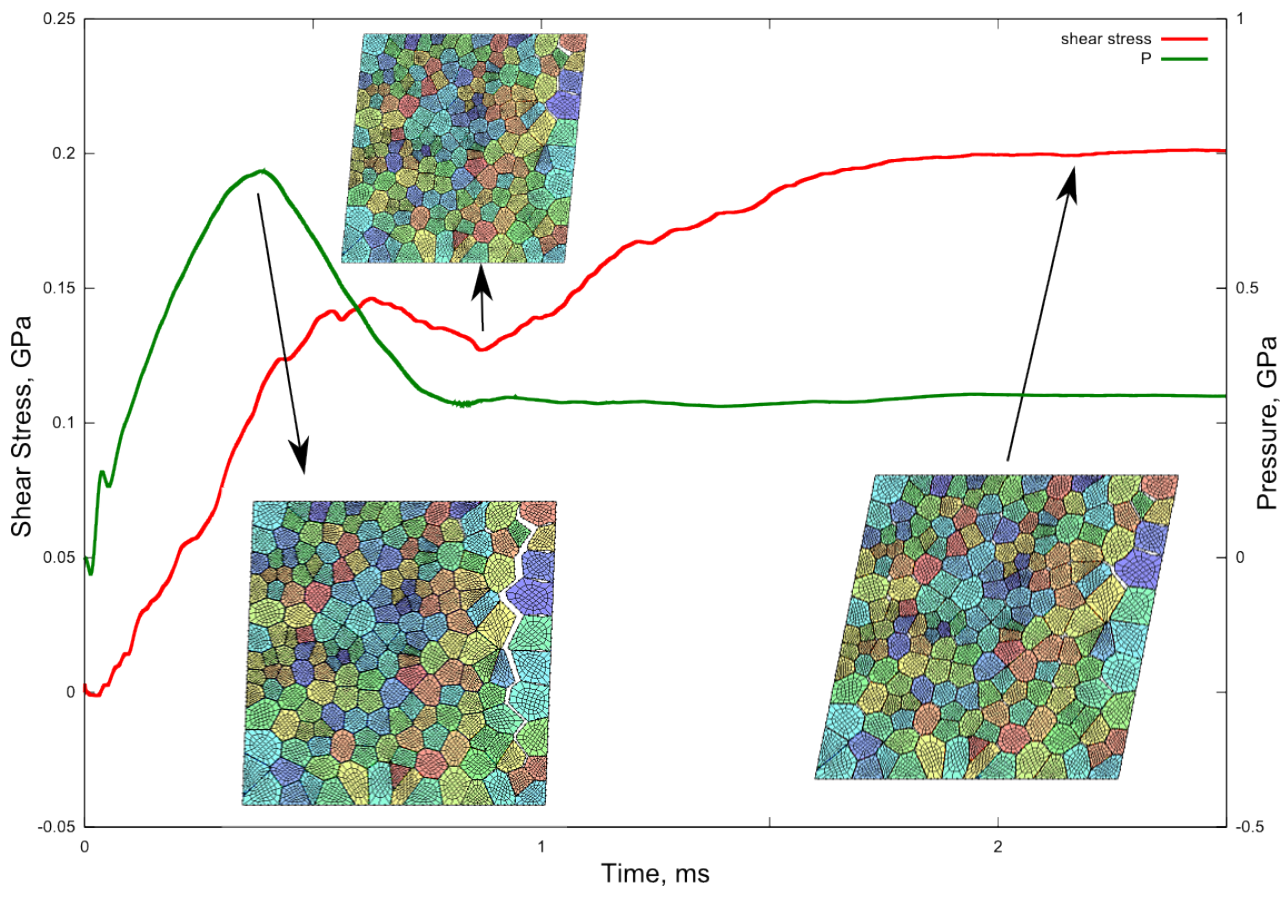

Figure 3.6: Shear stress and pressure evolution for 2D RVE made of packed blocks. Block deformations is shown at various times

few orders of magnitude less than the cell size. Therefore, the second, discrete, approach looks more attractive since it explicitly tracks deformations in the localization zones of failed material applying continuum modeling elsewhere. We hope that such a discrete-continuum approach can be useful in meso-scale modeling of fracture and fragmentation especially in the systems with preexisting discontinuities. We apply common plane contact [11] with history variables describing damage at the contacts which in the limit of stiff and strong contacts describe continuum and, once material brakes, transition to frictionless slide contacts. Contacts change type from type 1 to type 0 (collisional contacts) when such transitions take place. Figure 3.6 shows an example of shear loading of an RVE made of tightly packed polygonal blocks. The blocks were meshed using premeshed spheres which were placed inside the polygones and deformed radially to conform to the boundaries. Then an equipotential zoning was applied to improve the mesh inside the polygone. Velocity controlled boundaries were applied at the boundary nodes.

Such method limits the possible path for the fracture surfaces to the existing mesh lines which may create a mesh bias. Possible solution is to seed the centers of weakness in the material and use meshing technique which connects those centers (for example, Voronoi triangulations). Changing the mesh resolution in 


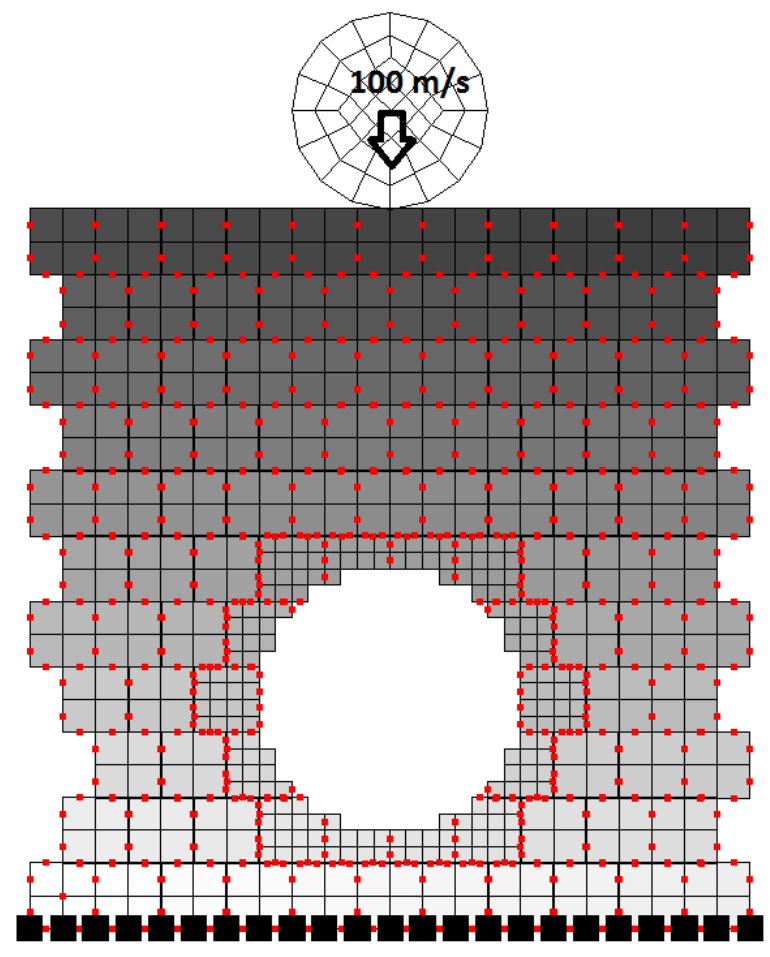

Figure 3.7: Cavity collapse under impact:Meshed blocks, contact boundaries between blocks(small dots) and fixed bottom boundary (large dots)

this case will guarantee that all meshes offer the same fracture paths aligned with the material weakness and, thus, less likely will be mesh sensitive.

\subsection{Dynamic element decoupling algorithm}

The alternative to a prefractured mesh described above is a dynamic element decoupling illustrated schematically in Figure 3.10. It has a few advantages over the prefractured algorithm described above especially if the failure is localized in a small area during the loading.

The following decoupling algorithm was implemented. Once the shear or tensile stress in an element reaches a critical value (within 10 percent to the failure criterion) this element is decoupled from the mesh and contact faces created for the existing faces of the element as well as new external faces which belong to the neighboring elements. The contact variables are initialized immediately to support both the normal and the shear stresses interpolated to the interface from the adjacent element centers. If high resolution is required to 

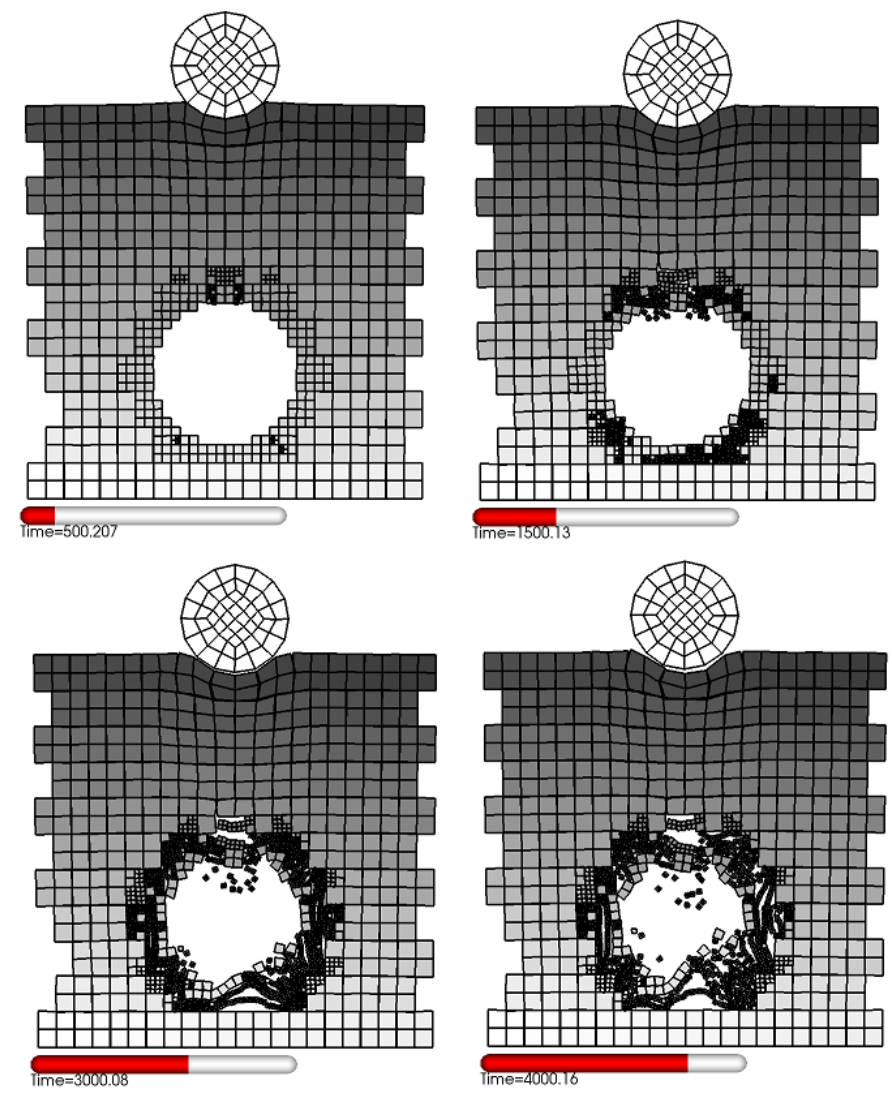

Figure 3.8: Cavity collapse under impact:block fracture and motion around the cavity at different times

resolve the fracture path withing the element, the element can be subdiscretized or remeshed. In this case, more than one external face is created for each side of the element which now becomes a subdiscretized mesh block. As it is seen from Figure 3.10, once an element is decoupled the damage starts evolving at the contacts leading to a complete separation from the continuum. Recursive decoupling algorithm was also implemented to help resolving smaller fragment sizes.

Figure 3.7 shows a simple 2D problem illustrating the dynamic decoupling algorithm with block refinement. A cylindrical tunnel in an imbricate wall is impacted by a steel cylindrical projectile with $100 \mathrm{~m} / \mathrm{s}$ velocity.

Figure. 3.8 shows how the cavity damage evolves during the calculations. Recursive decoupling helps to resolve various fragment sizes which could be compared with rubble size distribution measured in such experiments.

Another example of dynamic decoupling is shown in Figures 3.11. To model 


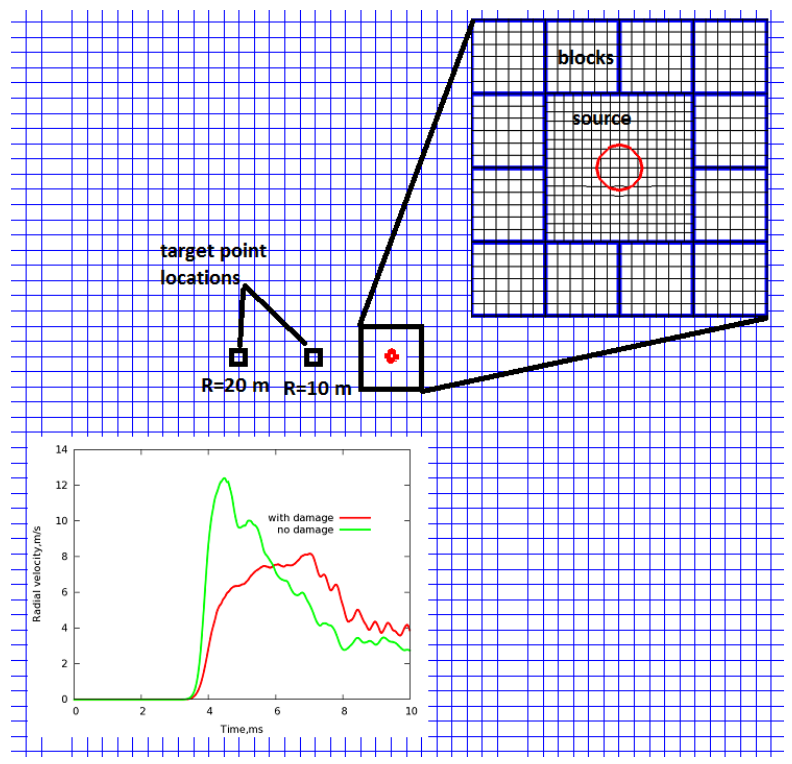

Figure 3.9: Block boundaries, target point location and computational mesh for $2 \mathrm{D}$ explosion in jointed rock. Radial velocity evolution at range $20 \mathrm{~m}$ is shown in the low left corner

explosion in a jointed rock with 2 perpendicular joint sets specific energy of 3.9 $\mathrm{kJ} / \mathrm{g}$ was deposited in a circular region in a bigger rock block as it is shown in Fig. 3.9. The wave propagating from the source caused some distortional damage generated at the contact interfaces between the decoupled elements. Initially the damage zone was symmetric, but later was guided by the two joint sets forming the shape of a cross aligned with the joint directions. Maximum shear slip at the joints was observed at 45 degrees to the joints.

\subsection{Combinig discrete and continuum methods to model wave propagation in a jointed rock formation}

Dynamic decoupling algorithm, DDA, described above has an important advantage over the XFEM methods used to represent cracks, joints, faults and other discontinuities in continuum simulations that it can handle large displacements and separations of blocks of rock including further contact dynamics between the blocks. This feature makes DDA absolutely essential to model collapse of buried structures. Yet applying contact detection for every single joint in the problem may be prohibitively expensive. We have partly addressed this by developing a smart hybrid cohesive-collisional contact described above. Yet, if deformations 


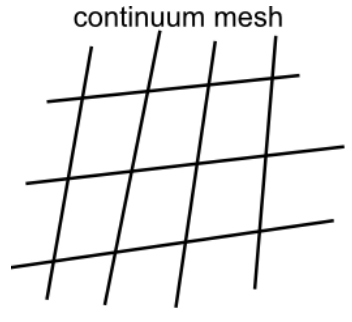

Time $=0$

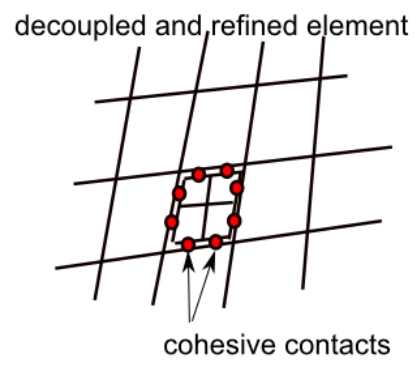

Time $=\mathrm{t} 1$

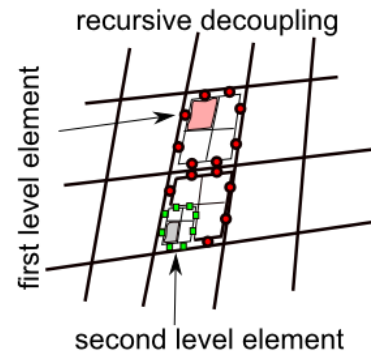

Time $=\mathrm{t} 2>\mathrm{t} 1$

Figure 3.10: Schematic representation of dynamic decoupling algorithm

on many contact surfaces remain small through the calculations it may be more efficient to use a continuum, implicit description of joints as weakness planes cutting through the elements. Details of this approach are described in [6]. It may not be always easy to determine where to use implicit description of joints, since large deformations may develop unexpectedly and cause mesh tangling of such elements. We have developed a method to transition to the discrete fracture using element decoupling algorithm once large deformations, which may cause element tangling, are developed. Results shown in Fig. (3.13,3.14,3.15) illustrate this combined discrete-continuum fracture approach. To model explosion in a jointed rock with 2 perpendicular joint sets with $10 \mathrm{~m}$ joint spacing specific energy of $3 . \mathrm{kJ} / \mathrm{g}$ was deposited in a circular region at depth $35 \mathrm{~m}$ as it is shown in Fig. 3.12.

\subsection{D application of element decoupling algo- rithm for dynamic tunnel collapse}

Developed decoupling algorithm can be applied for analysis of tunnel stability under dynamic loading caused by an explosion. To illustrate how this algorithm can be applied a berried explosion in a jointed granite rock formation with 3 joint sets was simulated as it is shown in Figure. 3.16. The source was modeled as an instantaneous energy deposition equivalent 180 tons of TNT withing 1 $\mathrm{m}$ ideal gas material at $7 \mathrm{~m}$ depth. The pressures of the order of few GPa are generated around the source which drop to a fraction of $1 \mathrm{GPa}$ when the wave reaches the tunnel located $30 \mathrm{~m}$ below the ground. The wave reflects from the walls of the tunnel causing some tensile damage. Continuum damage is used to trigger element decoupling around the tunnel. Figure. 3.17 shows evolution of the damage caused by the wave. As damage reaches a threshold value the elements are decoupled from the mesh and subdiscretized. Contacts are inserted between these elements which support both tension and shear but degrade as the 


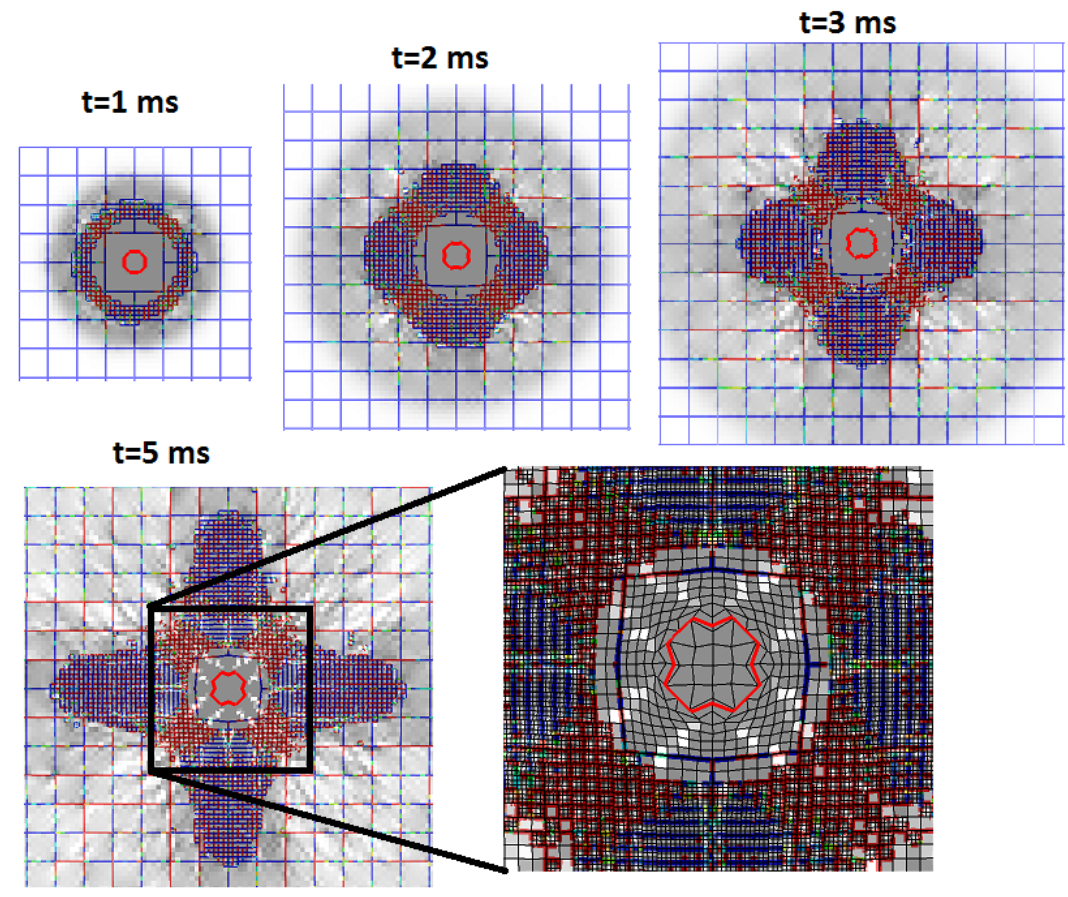

Figure 3.11: Pressure contours (0-0.1 GPa), shear slip at the joints (0-1) and material boundary between the source and rock. Enlarged source region is shown on the right

deformation at the contacts evolves due to external load. Thus, instead of using continuum model to model failure (which would require at least few elements across the failure zone) we transition into a discrete model. The new blocks can be decoupled further to evaluate the fragment size distribution for the rubble. Once the rubble blocks are formed they will fall into the tunnel due to the gravity effect. This would require a much longer calculation. Hybrid element solver has been developed to evaluate the kinematics of the rubble where a rigid body solver can be applied at later times. 


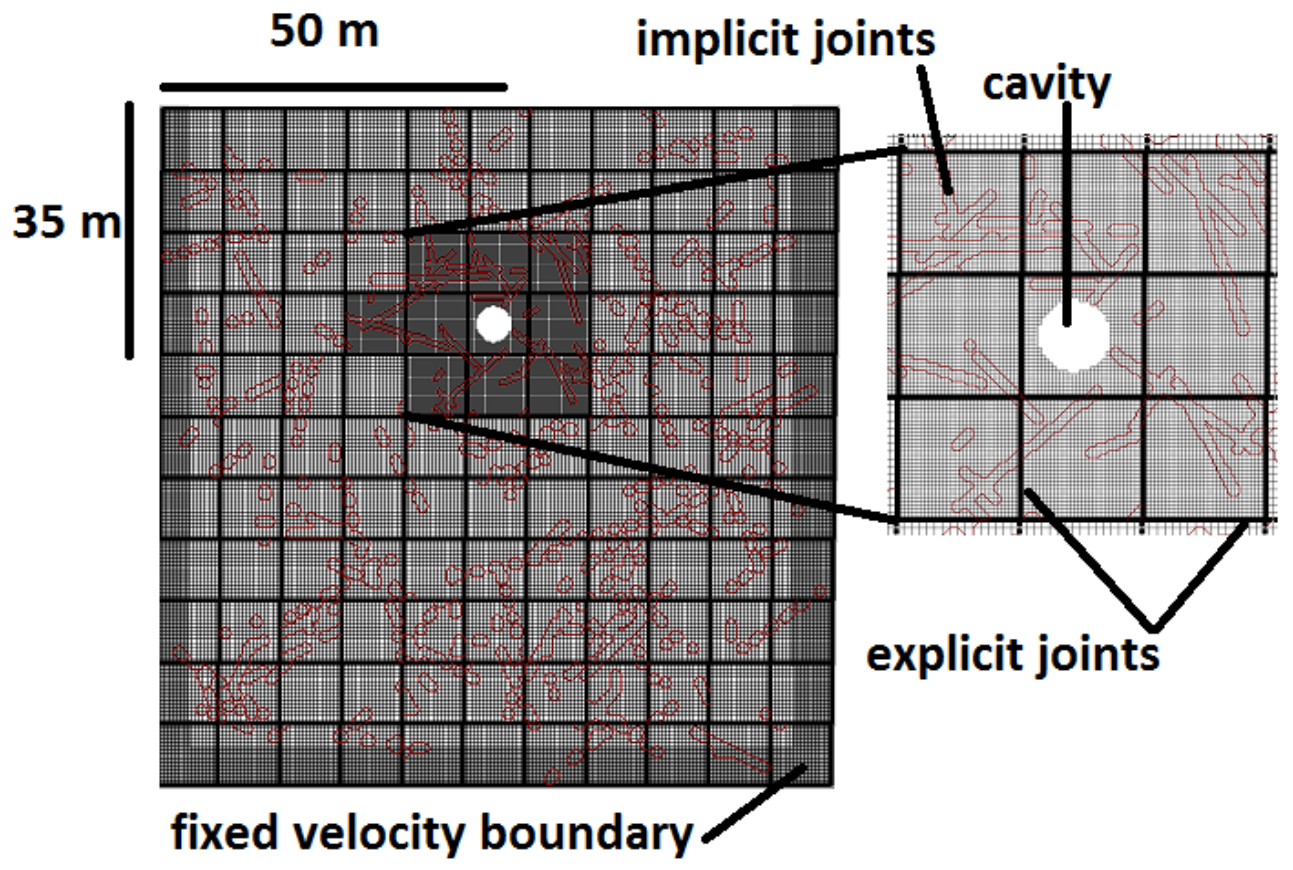

Figure 3.12: Computational domain for 2D explosion simulation. Squared rock blocks $10 \times 10 \mathrm{~m}$ were used 

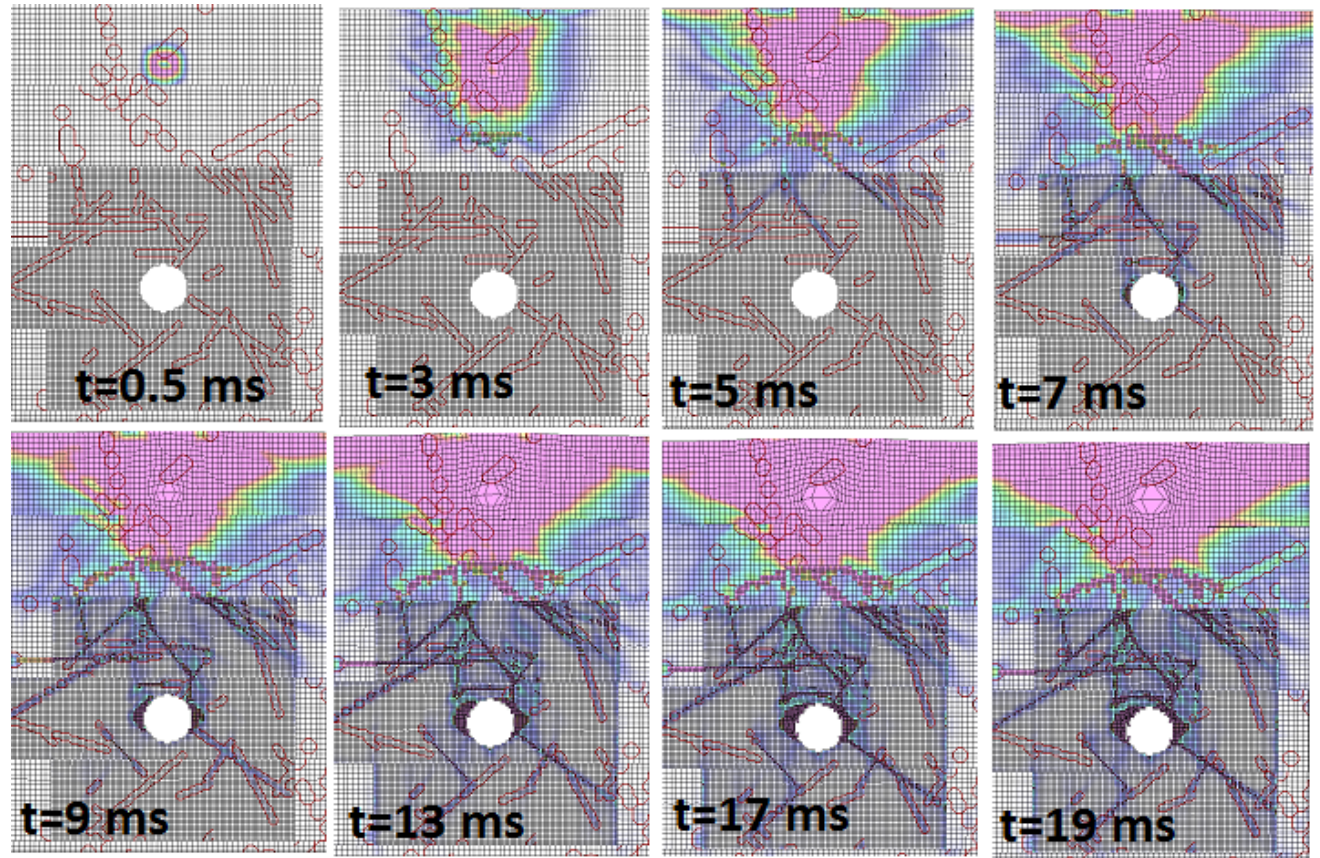

Figure 3.13: Plastic strain evolution (0-1\%) cased by the explosion for fully persistent rock mass (two sets) with extra random joints modeled as weakness planes 


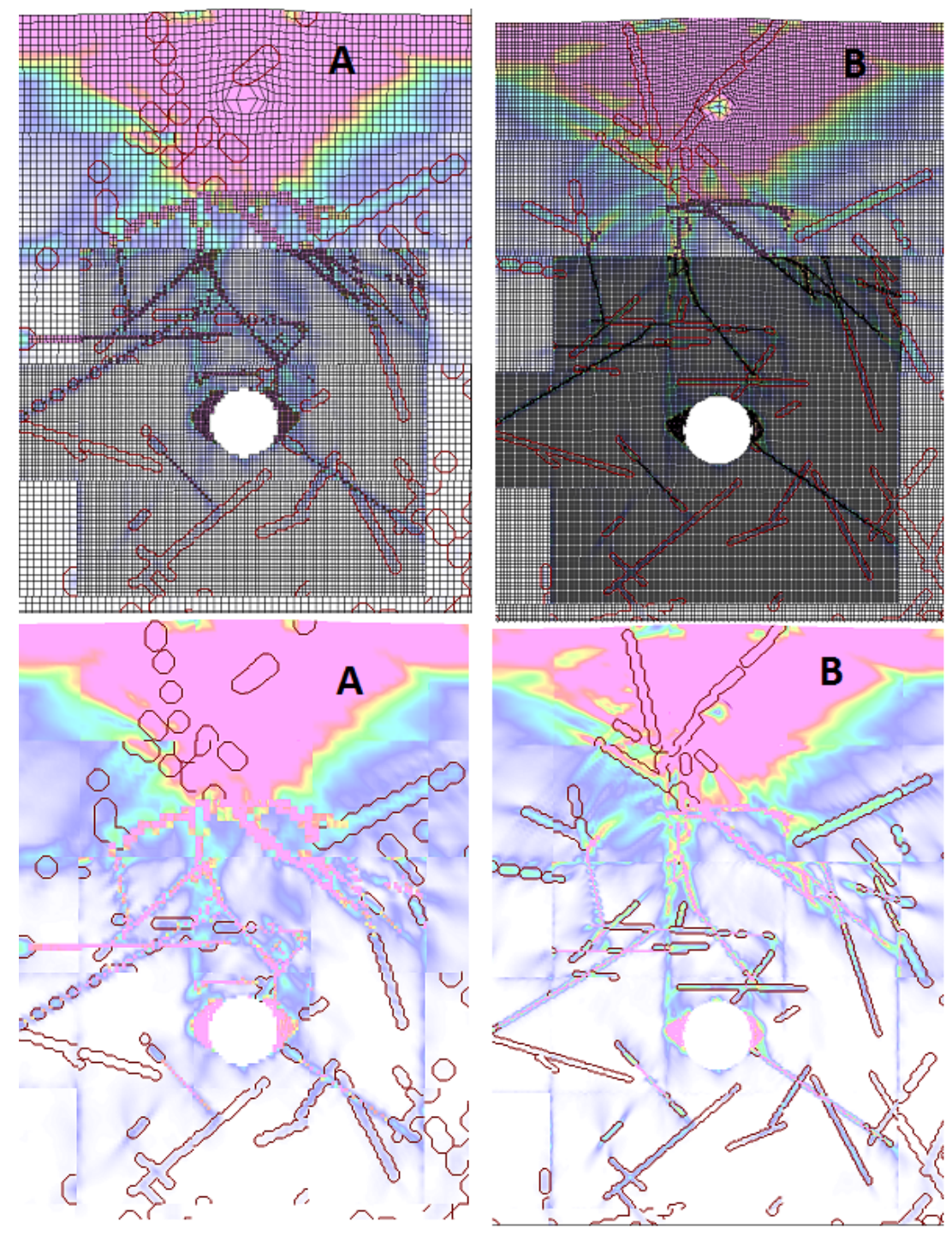

Figure 3.14: Comparing solutions at $11 \mathrm{~m}$ for a twice finer mesh 


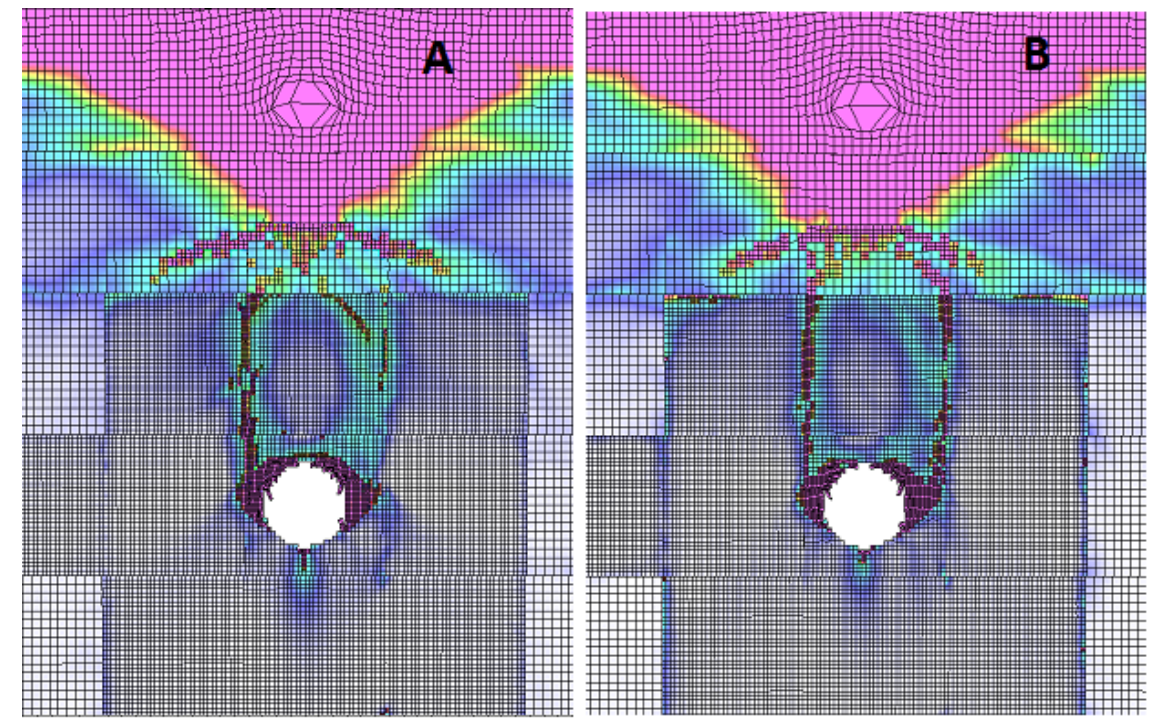

Figure 3.15: Comparing plastic strain contours at 19 ms where $100 \%$ and $50 \%$ persistent explicit joints

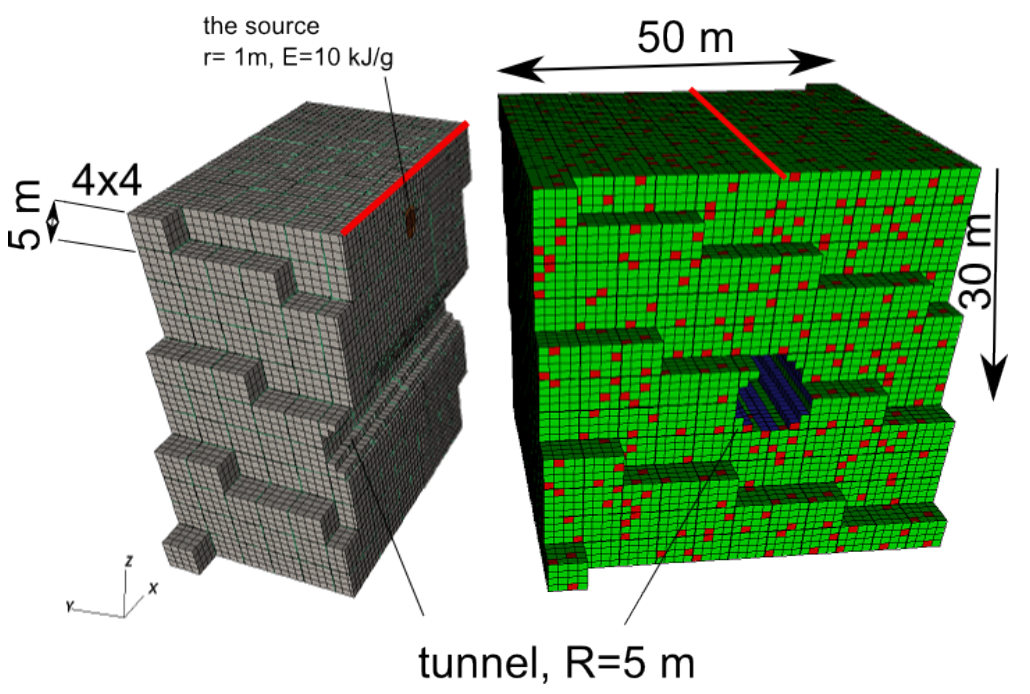

Figure 3.16: Computational domain for 3D explosion simulation. A crossection of the region along the tunnel is shown on the left 


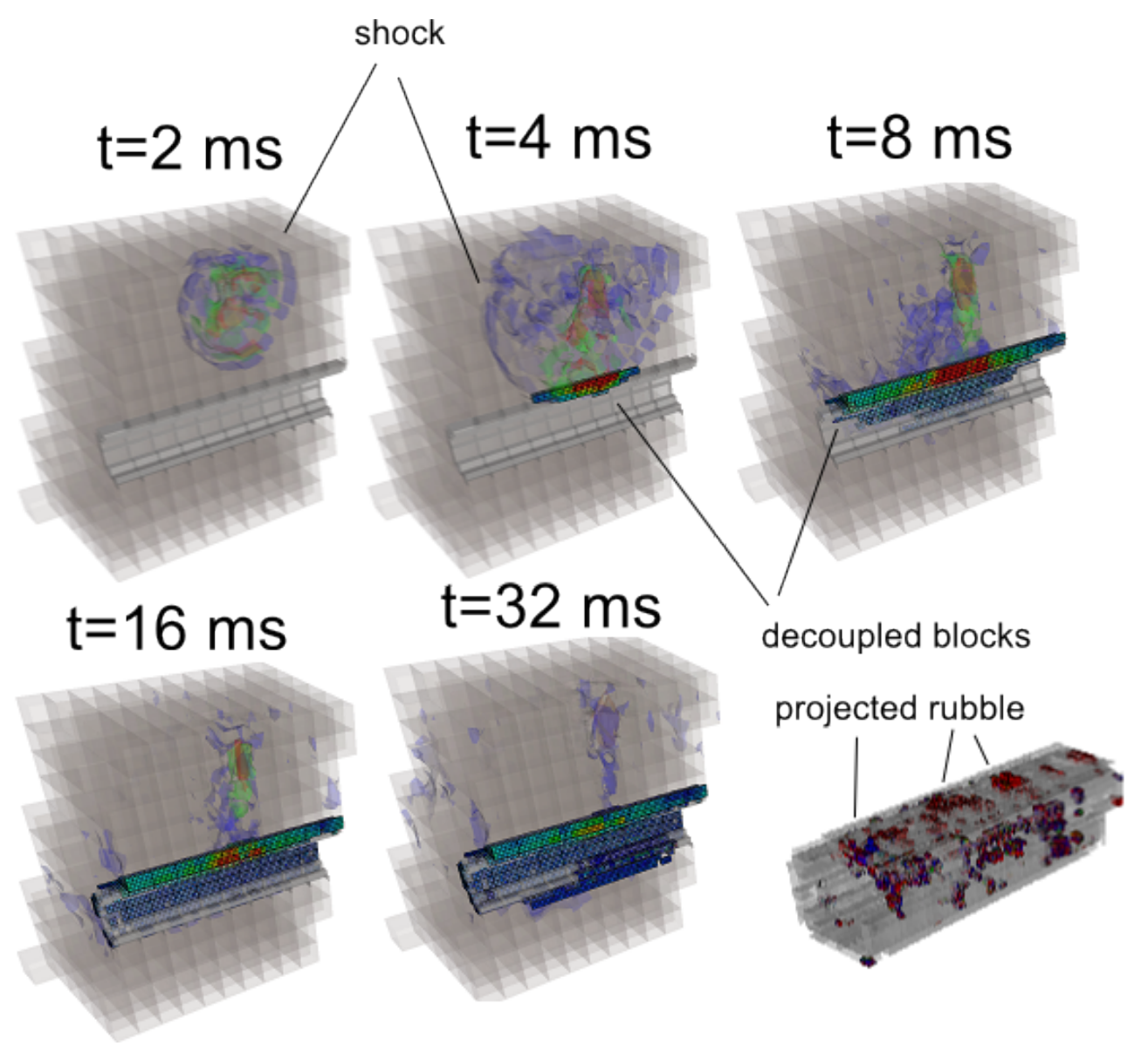

Figure 3.17: Contours of vertical stress $(0-0.1 \mathrm{GPa})$ and vertical velocity in damaged material around the tunnel at different times 


\section{Chapter 4}

\section{Validation and sensitivity study}

\subsection{Large scale model validations}

Before any sensitivity analysis can be performed the model has to be validated for a realistic geology and loading conditions. The model for granite has been validated for a series of undeground explosions conducted in Nevada. Thus simulations were performed using the insitu granite model to match velocity and displacement attenuation from HARDHAT, PILE DRIVER, and SHOAL in granitic rocks. Figure 4.1 shows peak velocity attenuation measured for these events vs calculations done with the insitu model.

Also, the model was calibrated for Source Physics Experiment (SPE) conducted in Nevada in 2011-2012. SPE-N is a series of chemical explosions in the Climax Stock granite. The site used for SPE was thoroughly characterized providing a unique opportunity to create a realistic computational model of the site and perform validation exersise. During SPE events a $1 \mathrm{t}$ source was detonated at $55 \mathrm{~m}$ depth. Details of this simulations can be found in [45] and recently in [46]. Jointed rock is anisotropic and therefore the waveforms change depending on asimuthal direction. That explains observed variations in calculated peak velocities which are comparable to ones measured in the experiments. The joints make the pulse much longer, similar to what was observed in the experiment. So, the joints effect the peak displacement much more than they effect the peak velocity. Using discrete simulations which match the peak velocities and displacements pretty well, a better inSitu granite model was built which can be applied in less expensive continuum calculations. 


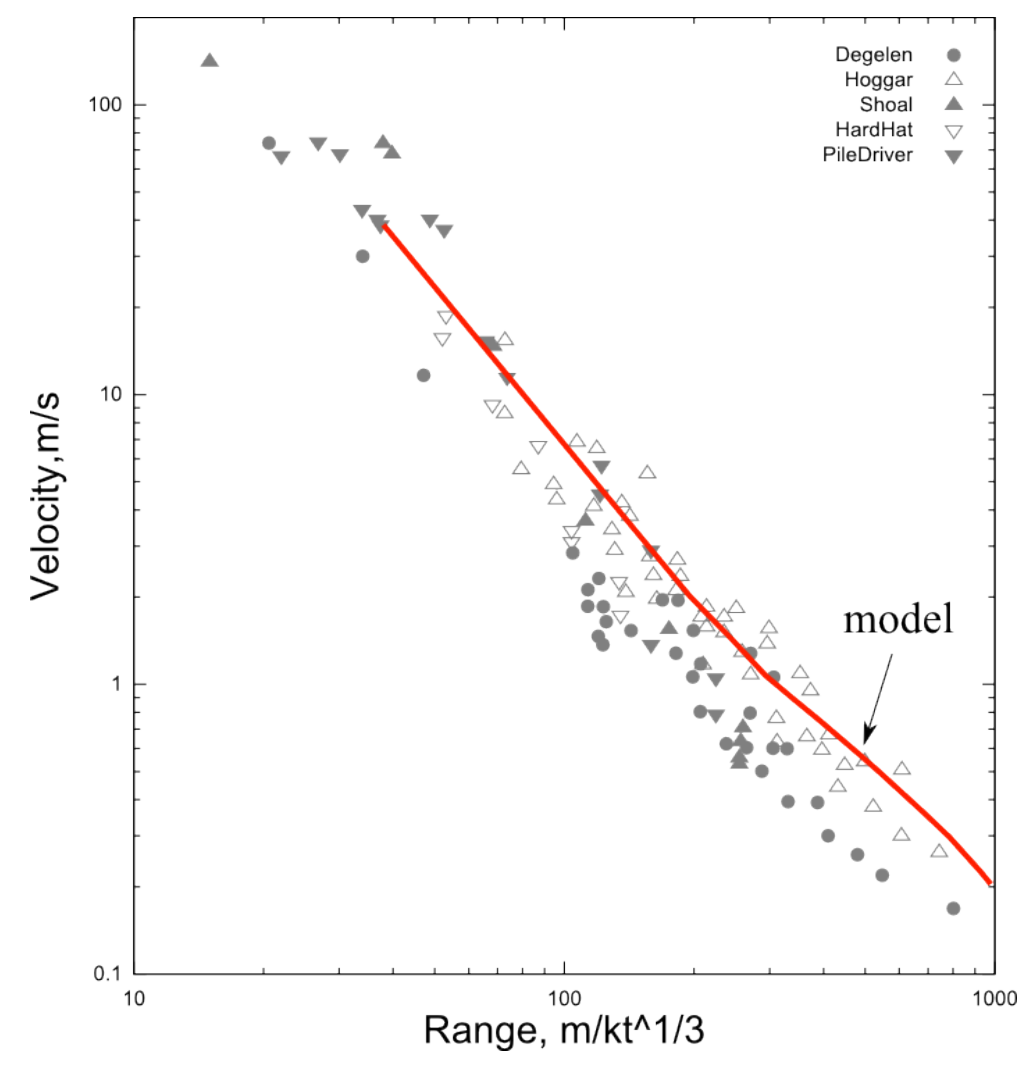

Figure 4.1: Peak velocity attenuations:measurements vs calculations

\subsection{Code coupling and discrete-continuum cal- culations}

Using Lagrangian codes is not the best way to model near-surface explosions because of the large material deformations in close vicinity to the source. Figure 4.2 illustrates a simple one-way coupling method that can be used to model the source in a Lagrangian code. The source can be modeled in a separate $2 \mathrm{D}$ axisymmetric calculation using an Eulerian code. The motion around the source is recorded and is used to enforce velocity boundary condition at the Lagrangian nodes located within a cylindrical region corresponding to the source. Largescale discrete simulations are performed with the joints modelled explicitly as contact interfaces. Then, at step A, information calculated in the discrete problem is used to develope a better continuum model which would match as much as possible the ground motion calculated in the discrete problem. Continuum calculations can be few orders of magnitude less expensive than the discrete ones since there is no need to track the motion of millions of joints which exist 
in the discrete model, and therefore they can be used in the analysis to evaluate the ground motion in various locations for different sources. The second step (step B) can be used further to study the stability of the structure to different loadings recorded in the large-scale continuum calculations. At this scale one may need to model discontinuities explicitly again, since they may effect the way how the structure would respond to the loading [4].

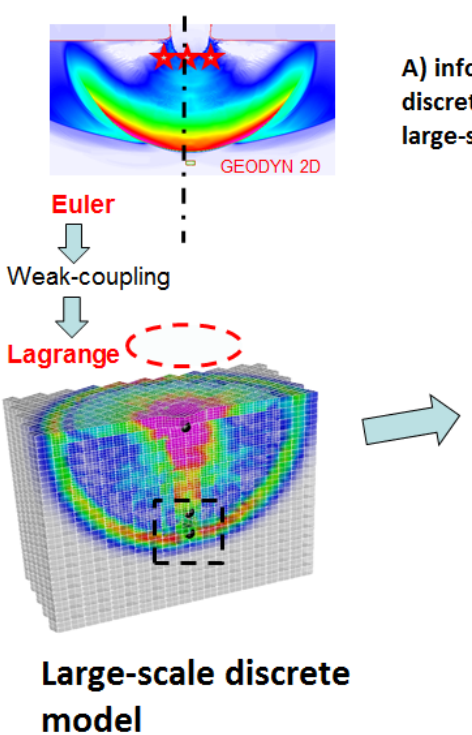

B) study structural

A) inform continuum model using response using loading discrete results and built a better histories recorded in large-scale continuum model large-scale calculations

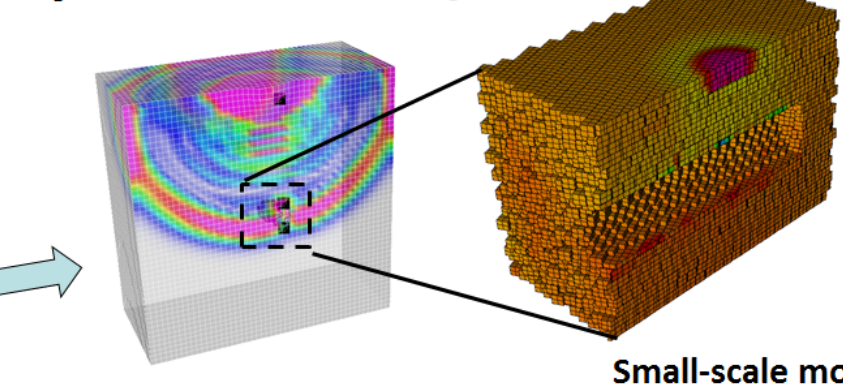
Large-scale continuum model

Small-scale model

Figure 4.2: Coupling technique used to study tunnel damage caused by a nearsurface explosion

\subsection{Wave focusing and shear motion caused by joints}

It has been known from undeground testing that spherical explosions can produce significant shear motions and wave anisotropy manifested in big variations of peak velocities and displacements in signals measured at the same range but in various azimuthal directions. SPE2/SPE3 experiments performed recently were not exceptions. To understand how joints can change a spherical wave we have conducted a study with a single joint set for a spherical explosion of the same yield as one in SPE3 experiments. Figure. 4.3 shows the problem set-up using axial symmetry. The motion was calculated at two sets of gauges A0-A90 located at the same ranges $(10$ and $20 \mathrm{~m})$ but at different angles relative to the joints set. Figure. 4.4 shows velocity vectors calculated at $7 \mathrm{~ms}$. Green lines at joints locations show plastic slip above certain threshold value. It is 
seen how the radial motion at 45 degrees is redirected in vertical and horizontal directions due to sliding at the joints. Joint spacing effect is shown in Fig. 4.5. A single joint (Fig. 4.5 case A ) creates two regions of non-radial motion, with clockwise shear motion behind the joints and counter clockwise motion infront of the joint. Since the joint begins to slip, when the wave incidence angle is less than the friction angle, the motion is redirected in vertical direction in front of the joints and in horizontal (normal to joint) direction behind the joint. The region of non-radial motion is wider when more joints are added (cases $\mathbf{A}$ and B)

Focusing effect of joints can be illustrated on a simple probelm of wave propagation of a surface blast to a tunnel burried underground as it is shown in fig. 4.7 Using validated granite model a series of $3 \mathrm{D}$ simulations has been performed to evaluate how persistency of joints can affect the wave attenuation in a rock formation withthree sets of joints shown in Fig. 4.8. Eulerian-Lagrangian code coupling described above was used to initialize the problem. Figure 4.9 shows that the joint persistency plays an important role in directing energy from the source. Waves tend to propagate in the directions either normal or perpendicular to the joint sets causing a significant anisotropy if the joints are persistent. When no joints were present, peak velocity generated at the top of the structure at depth $90 \mathrm{~m}$ below the surface was $18 \mathrm{~m} / \mathrm{s}$. For $95 \%$ persistent vertical joints the peak velocity reached $30 \mathrm{~m} / \mathrm{s}$. 


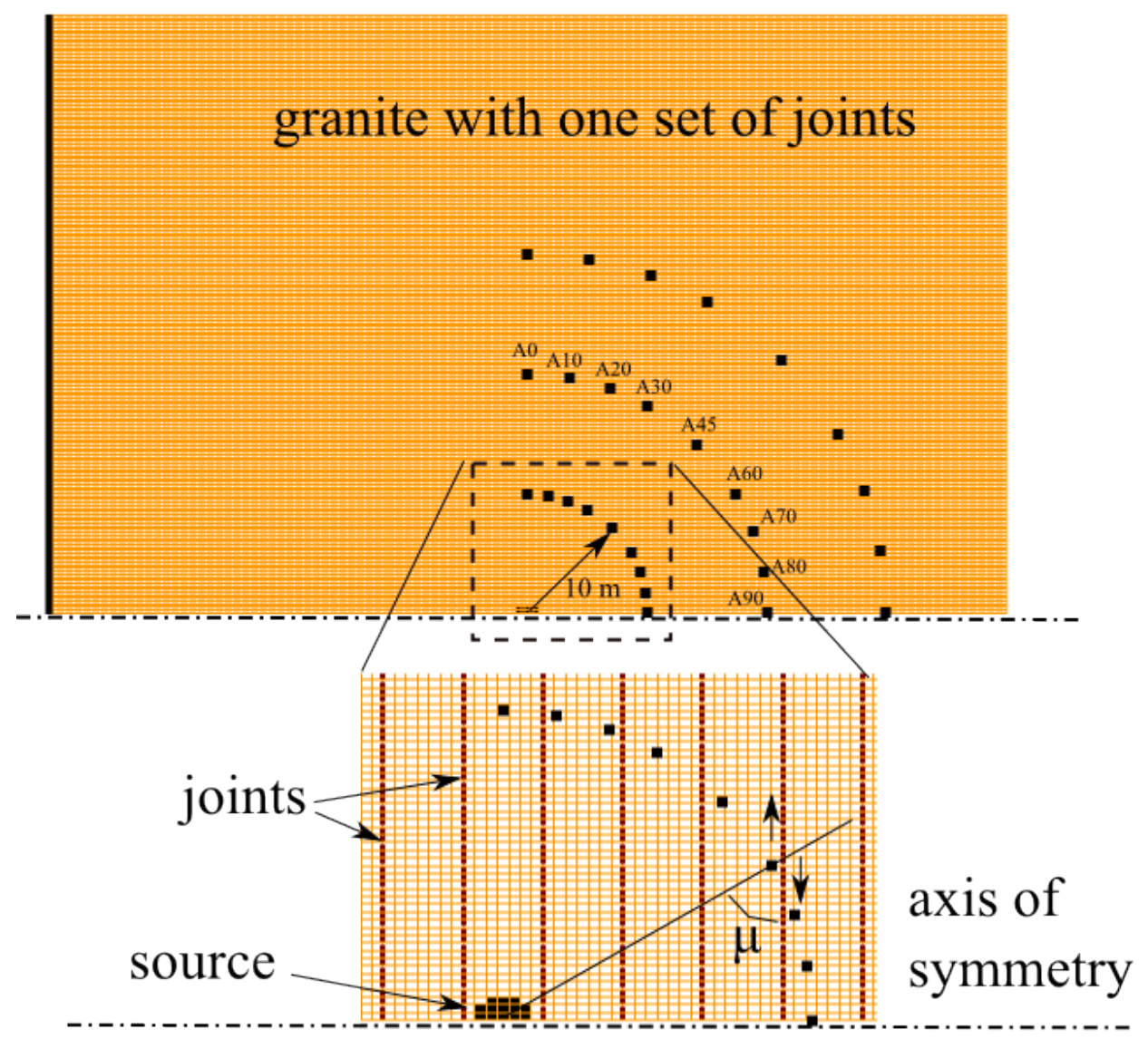

Figure 4.3: Computational mesh, location of joints and target points 


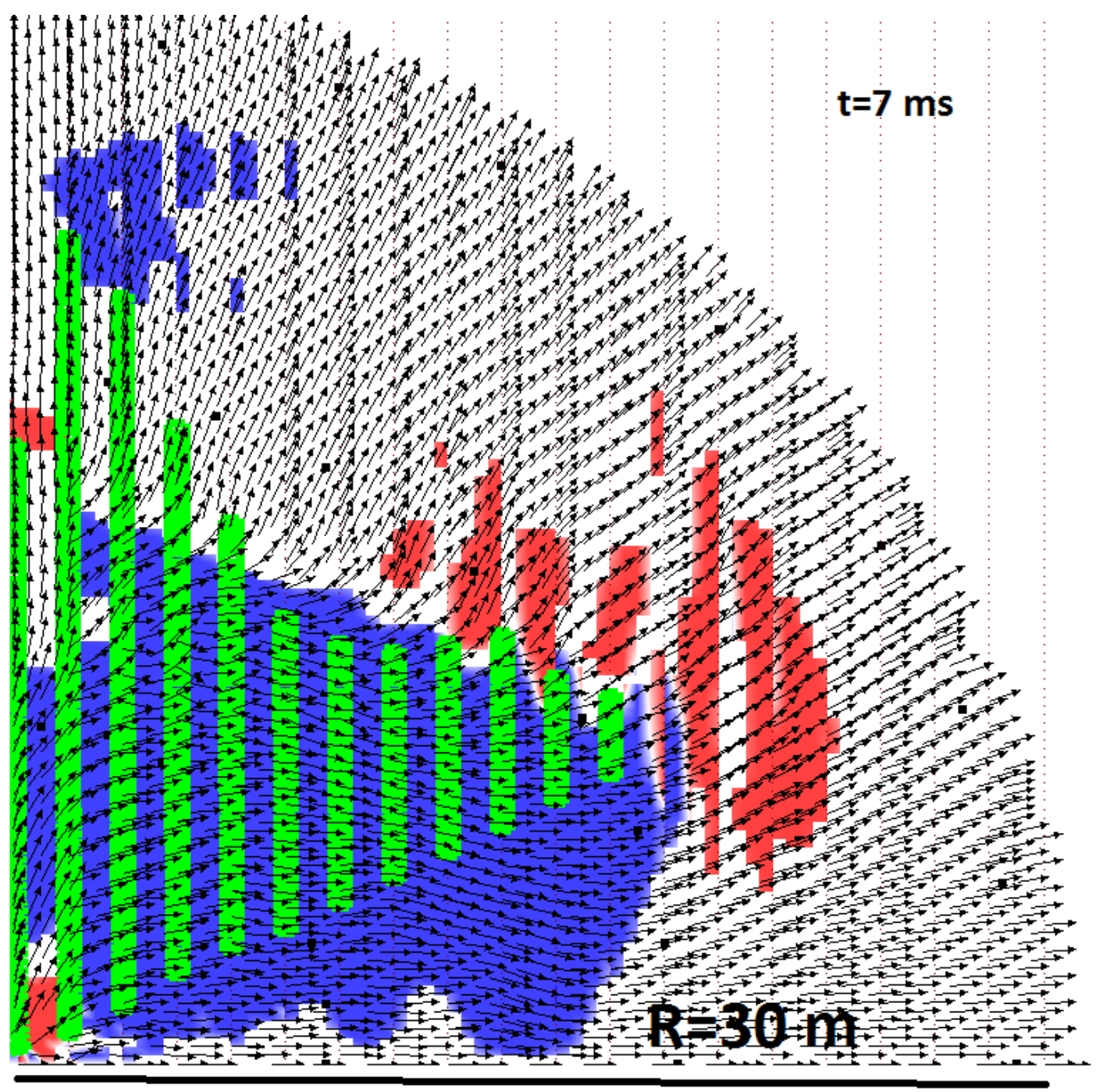

Figure 4.4: Redirection of radial motion from a spherical explosion by a single joint set 


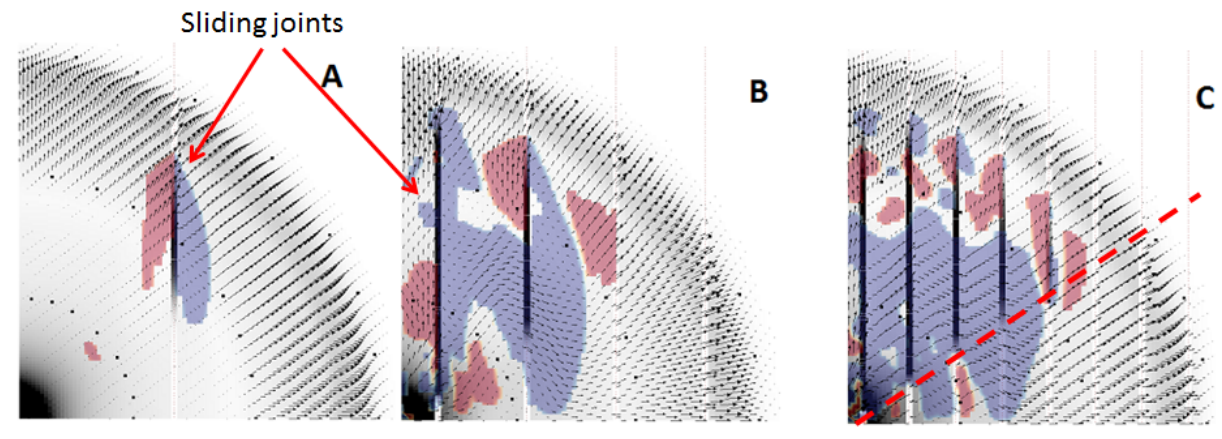

Figure 4.5: Effect of joint density on a flow caused by spherical explosion. Sliding joints are shown with dark vertical lines. Two contours (red and blue) show the regions with clock-wise (blue) and counter-clockwise motion. A- signle joint,Bsingle joint set, C-single joint set with decreased joint spacing where the dashed line corresponds to the friction angle at the joints.

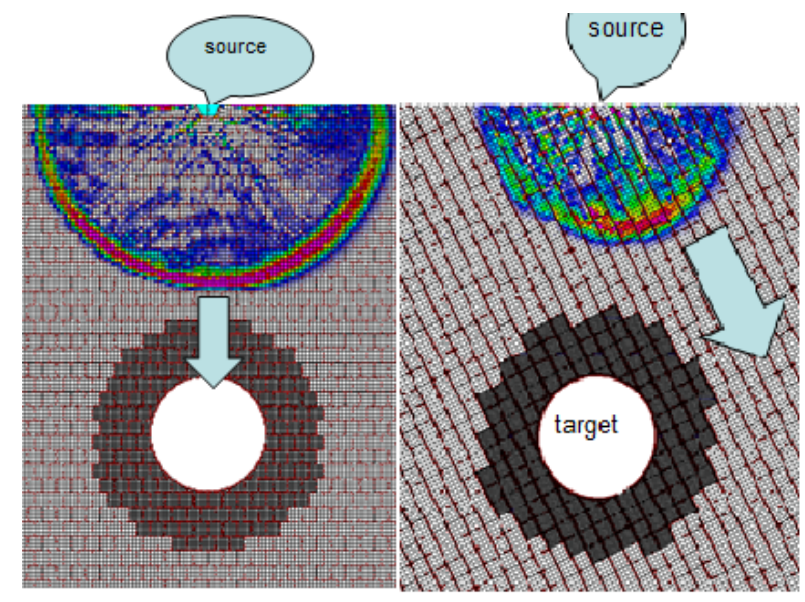

Figure 4.6: Example of oblique joint set guiding the shock wave away from the target 


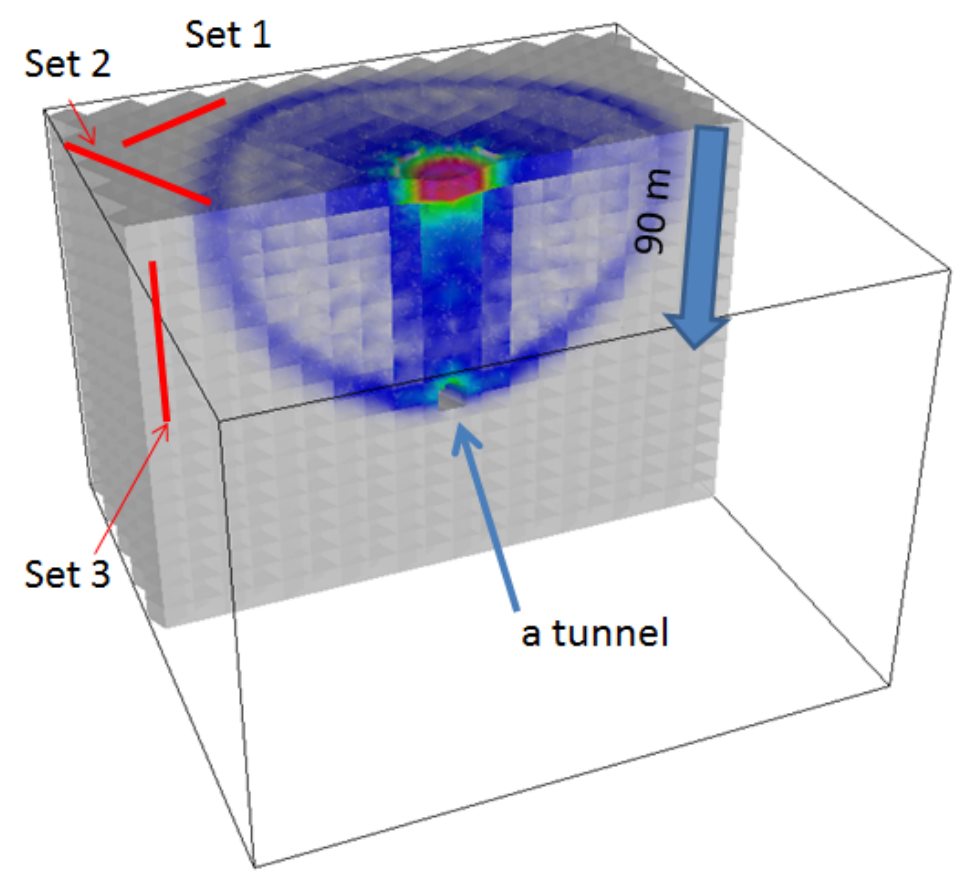

Figure 4.7: Joint orientation and contours of pressure wave due to surface explosion
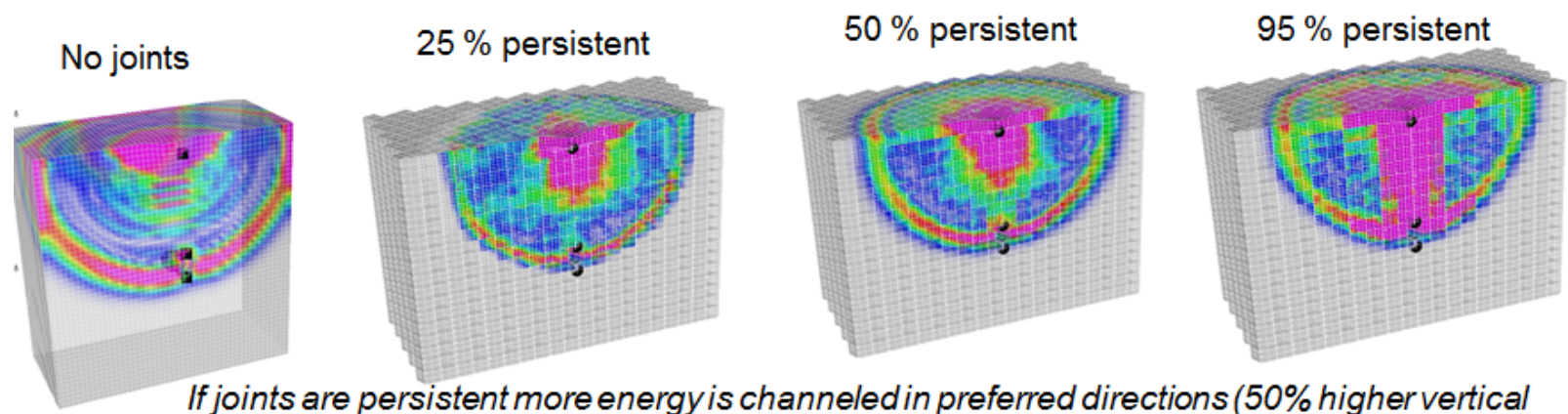
velocity for $95 \%$ persistent joints compared to no joints)

Figure 4.8: Persistency effect on wave generation in jointed granite formation 


\section{Chapter 5}

\section{Conclusion}

A new computational framework has been developed to model shock wave generation and propagation in a jointed rock formation. It includes a constitutive model capable to scale mechanical properties from the intact samples to jointed rock masses, a contact model with history variables capable of reproducing essential mechanical responses of rock joints as well as other discontinuities, and meshing techniques capable to create a numerical mesh for representative volumes of different scales (from RVE including characteristic jointed rock blocks used to study synthetic response of the rock mass and derive better continuum models for large scale, to field scale models including millions of joints of different properties used to model some large scale tests such as SPE). From numerical point of view, such a framework relied heavily on discrete-continuum computational techniques developed for massively parallel computations. The problems considered are so big, that they cannot fit in memory of the most powerful single CPU machines available today. Therefore, it was imperative to design the computational algorithms for a distributed memory computing using MPI communications both for calculations and data processing [11, 47].

\subsection{Main achievements of the project}

- Advanced discrete-continuum numerical techniques designed for massively parallel computations have been developed

- These methods have been applied to meso-scale study to understand mechanical response of jointed rock formation in the case of both quasi-static and dynamic loading

- Synthetic response of rock masses generated in these meso-scale studies was used to develop better continuum models for jointed rock masses.

- Improved continuum models for rock masses were used in large scale simulations to model shock wave propagation through jointed rock formations, 
while improved discrete methods, used before in meso-scale study, were used to study structure damage caused by the load

- Hybrid discrete-continuum method developed present a significant step forward both in computational mechanics and material science.

The results of this research have been presented at following scientific forums:

- IV European Congress on Computational Mechanics (ECCM IV), Paris,France, May 2010

- IWMM 2010, Stanford,USA, June 2010

- ARMA 2010, Salt Lake City,USA, July 2010

- IWPM 2010, Livermore,USA, September 2010

- EMI2011, Boston,USA, May 2011

- Plasticity 2012, Puerto-Rico,USA, January 2012

- 6th EUROPEAN CONGRESS ON COMPUTATIONAL METHODS IN APPLIED SCIENCES AND ENGINEERING (ECCOMAS 2012), Vienna, Austria, September 2012

The results of this work is described in the proceedings of the conferences listed above and published in the following peer-reviewed publications [6, 48, 11]

Two papers describing the latest achievements are under preparations for peer-reviewed publications.

\subsection{Benefits to other projects}

- The contact model developed within the current project was partly implemented in ALE3D code which benefited defense applications.

- The methods developed were used to model and understand Source Physics Experiments conducted under NNSA project which will benefit Nuclear Treaty Verification work.

- Meso-scale methods developed in the current study are used in the other projects where meso-scale response of discontinuous media is investigated. They will help to understand penetration resistance of various distinctly discrete targets such as sand and fragmented ceramic powder. 


\section{Bibliography}

[1] Senseny P, Pucik T. Development and validation of computer models for structures in jointed rock. INTERNATIONAL JOURNAL FOR NUMERICAL AND ANALYTICAL METHODS IN GEOMECHANICS JUL 1999; 23(8):751-778, doi:10.1002/(SICI)1096-9853(199907)23:8¡751::AIDNAG4¿3.0.CO;2-1.

[2] Burnett S, Gilbert M, Molyneaux T, Beattie G, Hobbs B. The performance of unreinforced masonry walls subjected to low-velocity impacts: Finite element analysis. INTERNATIONAL JOURNAL OF IMPACT ENGINEERING AUG 2007; 34(8):1433-1450, doi:10.1016/j.ijimpeng.2006.08.004.

[3] Massart T, Peerlings R, Geers M. Mesoscopic modeling of failure and damage-induced anisotropy in brick masonry. EUROPEAN JOURNAL OF MECHANICS A-SOLIDS SEP-OCT 2004; 23(5):719-735, doi: 10.1016/j.euromechsol.2004.05.003.

[4] Morris J, Rubin M, Blair S, Glenn L, Heuze F. Simulations of underground structures subjected to dynamic loading using the distinct element method. ENGINEERING COMPUTATIONS 2004; 21(2-4):384-408, doi: 10.1108/02644400410519848. 3rd International Conference on Discrete Element Methods, Santa Fe, NM, SEP, 2002.

[5] Hallquist J, Goudreau G, Benson D. Sliding interfaces with contact-impact in large-scale lagrangian computations. Computer Methods in Applied Mechanics and Engineering 1985; 51(1-3):107 - 137, doi:DOI: 10.1016/00457825(85)90030-1.

[6] Vorobiev O. Discrete and continuum methods for numerical simulations of non-linear wave propagation in discontinuous media. INTERNATIONAL JOURNAL FOR NUMERICAL METHODS IN ENGINEERING JUL 23 2010; 83(4):482-507, doi:10.1002/nme.2840.

[7] Pine R, Owen D, Coggan J, Rance J. A new discrete fracture modelling approach for rock masses. Geotechnique 2007; 57(9):757-766.

[8] Mas Ivars D, Pierce ME, Darcel C, Reyes-Montes J, Potyondy DO, Paul Young R, Cundall PA. The synthetic rock mass approach for jointed 
rock mass modelling. International Journal of Rock Mechanics and Mining Sciences 2011; 48(2):219-244.

[9] Kawa M, Pietruszczak S, Shieh-Beygi B. Limit states for brick masonry based on homogenization approach. International Journal of Solids and Structures 2008; 45(34):998 - 1016, doi:10.1016/j.ijsolstr.2007.09.015. URL http://www.sciencedirect.com/science/article/pii/S0020768307003800.

[10] Uva G, Salerno G. Towards a multiscale analysis of periodic masonry brickwork: A fem algorithm with damage and friction. International Journal of Solids and Structures 2006; 43(13):3739 - 3769, doi:10.1016/j.ijsolstr.2005.10.004. URL http://www.sciencedirect.com/science/article/pii/S0020768305005767.

[11] Vorobiev O. Simple Common Plane contact algorithm. INTERNATIONAL JOURNAL FOR NUMERICAL METHODS IN ENGINEERING APR 13 2012; 90(2):243-268, doi:10.1002/nme.3324.

[12] Vorobiev O. Generic strength model for dry jointed rock masses. International Journal of Plasticity 2008; 24(12):2221 - 2247, doi:DOI: 10.1016/j.ijplas.2008.06.009.

[13] Baud P, Zhu W, Wong Tf. Failure mode and weakening effect of water on sandstone. J. Geophys. Res. 2000; 105(B7):16 371 - 16389.

[14] Mowar S, Zaman M, Stearns D, Roegiers JC. Micro-mechanisms of pore collapse in limestone. Journal of Petroleum Science and Engineering 1996; 15(24):221 - 235, doi:10.1016/0920-4105(95)00065-8.

[15] Coelho LC, Soares AC, Ebecken NFF, Drummond Alves JL, Landau L. Modelling mechanical behaviour of limestone under reservoir conditions. International Journal for Numerical and Analytical Methods in Geomechanics 2006; 30(14):1477-1500, doi:10.1002/nag.543. URL http://dx.doi.org/10.1002/nag. 543 .

[16] Lomov I, Vorobiev O, Antoun TH. Simulation of shock wave propagation and damage in geologic materials. UCRL-CONF-206632, 2004.

[17] Zhang J, Wong TF, Yanagidani T, Davis DM. Pressure-induced microcracking and grain crushing in berea and boise sandstones: acoustic emission and quantitative microscopy measurements. Mechanics of Materials 1990; 9(1):1 - 15, doi:10.1016/0167-6636(90)90026-C.

[18] Larson D, Anderson G. Plane shock wave studies of westerly granite and nugget sandstone. International Journal of Rock Mechanics and Mining Sciences 83 Geomechanics Abstracts, vol. 17, Elsevier, 1980; 357-363.

[19] Schock R, Abey A, Bonner B, Duba A, Heard H. Mechanical properties of nugget sandstone. Technical Report, California Univ., Livermore (USA). Lawrence Livermore Lab. 1973. 
[20] Ma G, Hao H, Zhou Y. Modeling of wave propagation induced by underground explosion. Computers and Geotechnics 1998; 22(34):283 - 303, doi:10.1016/S0266-352X(98)00011-1.

[21] Murphy JR, Kitov IO, Rimer N, Adushkin VV, Barker BW. Seismic characteristics of cavity decoupled explosions in limestone: An analysis of soviet high explosive test data. J. Geophys. Res. 1997; 102(B12):27 393-27 405.

[22] Rubin M, Elata D, Attia A. Modeling added compressibility of porosity and the thermomechanical response of wet porous rock with application to mt. helen tuff. International Journal of Solids and Structures 1996; 33(6):761 - 793, doi:10.1016/0020-7683(95)00077-N.

[23] Rubin MB, Vorobiev OY, Glenn LA. Mechanical and numerical modeling of a porous elastic-viscoplastic material with tensile failure. International Journal of Solids and Structures 2000; 37(13):1841 - 1871, doi:DOI: 10.1016/S0020-7683(98)00333-3.

[24] Shao J, Henry J. Development of an elastoplastic model for porous rock. International Journal of Plasticity 1991; 7(12):1 - 13, doi:10.1016/07496419(91)90002-G.

[25] Xie S, Shao J. Elastoplastic deformation of a porous rock and water interaction. International Journal of Plasticity 2006; 22(12):2195 - 2225, doi:10.1016/j.ijplas.2006.03.002.

[26] Walsh JB. The effect of cracks on the compressibility of rock. J. Geophys. Res. 1965; 70(2):381-389.

[27] Vajdova V, Baud P, Wong Tf. Compaction, dilatancy, and failure in porous carbonate rocks. J. Geophys. Res. 2004; 109(B5).

[28] Vorobiev O, Liu B, Lomov I, Antoun T. Simulation of penetration into porous geologic media. International Journal of Impact Engineering 2007; 34(4):721 - 731, doi:DOI: 10.1016/j.ijimpeng.2006.02.002.

[29] Wong Tf, David C, Zhu W. The transition from brittle faulting to cataclastic flow in porous sandstones: Mechanical deformation. J. Geophys. Res. 1997; 102(B2):3009-3025.

[30] Mogi K. Fracture and flow of rocks under high triaxial compression. Journal of Geophysical Research 1971; 76(5):1255-1269.

[31] Mogi K. On the pressure dependence of strength of rocks and the coulomb fracture criterion. Tectonophysics 1974; 21(3):273-285.

[32] Hoek E, Brown E. Practical estimates of rock mass strength. International Journal of Rock Mechanics and Mining Sciences 1997; 34(8):1165-1186. 
[33] Hoek E, Carranza-Torres C, Corkum B. Hoek-brown failure criterion-2002 edition. 5th North American Rock Mechanics Symposium and 17th Tunneling Association of Canada Conference: NARMS-TAC, 2002; 267-271.

[34] Baud P, Schubnel A, Wong Tf. Dilatancy, compaction, and failure mode in solnhofen limestone. J. Geophys. Res. 2000; 105(B8):19 289-19 303.

[35] Török Á. Influence of fabric on the physical properties of limestones. Fracture and Failure of Natural Building Stones, Springer, Dordrecht 2006; :487-495.

[36] Chang C, Zoback MD, Khaksar A. Empirical relations between rock strength and physical properties in sedimentary rocks. Journal of Petroleum Science and Engineering 2006; 51(34):223 - 237, doi: 10.1016/j.petrol.2006.01.003.

[37] Sellgren U, Bjrklund S, Andersson S. A finite element-based model of normal contact between rough surfaces. Wear 2003; 254(11):1180 - 1188, doi: DOI: 10.1016/S0043-1648(03)00332-6. Papers presented at the 10th Nordic Conference on Tribology, NORDTRIB 2002.

[38] Sevostianov I, Kachanov M. Normal and tangential compliances of interface of rough surfaces with contacts of elliptic shape. International Journal of Solids and Structures 2008; 45(9):2723 - 2736, doi:DOI: 10.1016/j.ijsolstr.2007.12.024.

[39] Tremblay D, Simon R, Aubertin M. A constitutive model to predict the hydromechanical behaviour of rock joints. 60th Canadian Geotechnical Conference Ottawa, 2007; :2011-2018.

[40] Bandis S, Lumsden A, Barton N. Fundamentals of rock joint deformation. International Journal of Rock Mechanics and Mining Sciences Geomechanics Abstracts 1983; 20(6):249 - 268, doi:DOI: 10.1016/0148-9062(83)905958.

[41] Desai C, Fishman K. Plasticity-based constitutive model with associated testing for joints. International Journal of Rock Mechanics and Mining Sciences \& Geomechanics Abstracts 1991; 28(1):15 - 26, doi:DOI: 10.1016/0148-9062(91)93229-Y.

[42] Morris J. Review of rock joint models. Technical Report UCR-ID153650, Lawrence Livermore National Laboratories 2003, doi:DOI: $10.2172 / 15005881$.

[43] Fossum A. Effective elastic properties for a randomly jointed rock mass. International Journal of Rock Mechanics and Mining Sciences Geomechanics Abstracts 1985; 22(6):467 - 470, doi:DOI: 10.1016/0148-9062(85)90011-7.

[44] Cha M, Cho GC, Santamarina JC. Long-wavelength p-wave and s-wave propagation in jointed rock masses. Geophysics 2009; 74(5):E205-E214. 
[45] Antoun T, Xu H, Vorobiev O, Lomov I. Near field modeling of spe1 experiment and prediction of the second source physics experiments (spe2). Technical Report, Lawrence Livermore National Laboratory (LLNL), Livermore, CA 2011.

[46] Mellors R, Pitarka A, Rodgers A, Walter W, Ford S, Xu H, Matzel E, Myers S, Petersson N, Sjogreen B, et al.. Pre-shot simulations of near-field and far-field ground motion for the source physics experiment (spe) explosions at the climax stock, nevada national security site: Spe3. Technical Report, Lawrence Livermore National Laboratory (LLNL), Livermore, CA 2012.

[47] Wang F, Feng Y, Owen D. Parallelisation for finite-discrete element analysis in a distributed-memory environment. International Journal of Computational Engineering Science 2004; 5(01):1-23.

[48] Vorobiev O, Antoun T. Equivalent continuum modeling for non-linear wave propagation in jointed media. INTERNATIONAL JOURNAL FOR NUMERICAL METHODS IN ENGINEERING JUN 3 2011; 86(9):1101-1124, doi:10.1002/nme.3094. 